\title{
Interference of two co-directional exclusion processes in the presence of a static bottleneck: a biologically motivated model
}

\author{
Bhavya Mishra and Debashish Chowdhury \\ Department of Physics, Indian Institute of Technology Kanpur, 208016, India
}

\begin{abstract}
We develope a two-species exclusion process with a distinct pair of entry and exit sites for each species of rigid rods. The relatively slower forward stepping of the rods in an extended bottleneck region, located in between the two entry sites, controls the extent of interference of the co-directional flow of the two species of rods. The relative positions of the sites of entry of the two species of rods with respect to the location of the bottleneck are motivated by a biological phenomenon. However, the primary focus of the study here is to explore the effects of the interference of the flow of the two species of rods on their spatio-temporal organization and the regulations of this interference by the extended bottleneck. By a combination of mean-field theory and computer simulation we calculate the flux of both species of rods and their density profiles as well as the composite phase diagrams of the system. If the bottleneck is sufficiently stringent some of the phases become practically unrealizable although not ruled out on the basis of any fundamental physical principle. Moreover the extent of suppression of flow of the downstream entrants by the flow of the upstream entrants can also be regulated by the strength of the bottleneck. We speculate on the possible implications of the results in the context of the biological phenomenon that motivated the formulation of the theoretical model.
\end{abstract}

\section{INTRODUCTION}

Non-equilibrium steady states (NESS) of systems of interacting driven particles are of current interest in statistical physics [1, 2]. Totally asymmetric simple exclusion process (TASEP) $3 / 5$ is a paradigmatic model of interacting self-propelled particles $[2] 6$. In this model a fraction of the sites on a one-dimensional lattice are occupied by particles that can hop forward probabilistically, at a given rate (i.e., with a probability per unit time), if and only if its target site is empty. Various adaptations and extensions of TASEP, including multi-species exclusion processes 7455 , have been used to model vehicular traffic (see [7, 8, for reviews) and traffic-like collective phenomena in biological systems (see ref. 912 for reviews). Under open boundary conditions, the rates of entry and exit of the particles are also specified. In analogy with vehicular traffic, the points of entry and exit of the particles are often also referred to as the ON- and OFF- ramps, respectively. By convention adopted in this paper, all the particles move from left to right.

Special sites from which the particles can hop forward at a relatively slower rate are usually referred to as defect sites. An isolated slow site is a "point-like" defect whereas a continuous string of slow sites constitute a "line-like" (or "extended") defect. Each of these defects, irrespective of its size, creates a bottleneck where the flow of particles slows down. The effects of a single bottleneck as well as those of randomly distributed bottlenecks on the spatio-temporal organization of the particles, particularly the flow in the NESS, have been investigated over the last two decades [56 92. In all those models with bottlenecks only a single ON-, OFF-ramp pair was considered.

In this paper we develop a biologically motivated twospecies exclusion process with a distinct pair of $\mathrm{ON}$, OFF-ramps for each species, where an extended defect lies in between the two ON ramps. More specifically, the ON-ramp of one of the two species of particles is located immediately downstream from the right edge of a single extended bottleneck while the other ON ramp lies far upstream from the left edge of that defect.

Although the relative positions of the two ON-ramps with respect to the extended bottleneck in this model is motivated by a specific biological phenomenon, it is not intended to account for experimental data. Instead, the model focuses on the physics of collective spatio-temporal organization of the two species of particles. By influencing the flow of the particles that enter through the upstream ON-ramp, the bottleneck can control the extent of interference of the flow the two species in the downstream region. For this model, by a combination of mean-field theory and computer simulations, we draw the phase diagrams that displays an unprecedented richness. We also demonstrate a switch-like regulation of flow of one species of rods by a sufficiently high flow of the other.

\section{MODEL}

In the first two subsections we present brief summaries of TASEP with hard rods and the biological phenomenon of Internal Ribosome Entry Site (IRES) that motivates the model introduced in this paper.

\section{A. Brief summary of TASEP with hard rods and models of ribosome traffic}

In a TASEP under open boundary conditions equispaced sites on a one-dimensional lattice are labeled by the integer index $j=1,2, \ldots, L+\ell-1$. In the case TASEP with a single species of hard rods, each of length $\ell$ (in the units of lattice spacing), successive $\ell$ sites are cov- 
ered simultaneously by each rod. The position of each rod on the lattice is denoted by the lattice site covered by its leftmost edge. In our terminology used throughout this paper, a site $j$ is said to be occupied by a rod if $j$ denotes its position. Thus, at any arbitrary instant of time, if a site $j$ is occupied by a rod then all the sites $j, j+1, j+2, \ldots, j+\ell-1$ are simultaneously covered by the same rod. Thus, out of the $L+\ell-1$ lattice sites only the $L$ sites $1,2, \ldots, L$ can be occupied by the rods; the remaining $\ell-1$ sites can be covered by a rod if it is occupies the site $L$.

Mutual exclusion of the rods is ensured by imposing the condition that no lattice site can be covered by more than one rod simultaneously. At any arbitrary instant of time the number density of the rods on the lattice is defined by

$$
\rho=N / L
$$

where $N$ is the total number of rods occupying lattice sites at that instant of time. Since each rod simultaneously covers $\ell$ sites on the lattice and since none of the sites can be covered by more than one rod simultaneously the coverage density is given by

$$
\rho_{c}=\rho \ell
$$

where the corresponding number density $\rho$ is obtained from eq.(1). In other words, coverage density is the true measure of the total fraction of lattice sites occluded by the rods while only the remaining fraction remains empty.

Under open boundary conditions, a rod can enter the lattice only through the site $i=1$. If simultaneously all the first $\ell$ sites of the lattice (i.e., the sites $1,2, \ldots, \ell$ ) are not covered by any existing rod a new rod can enter the system and occupy the site $i=1$ (and also cover the sites $2, \ldots, \ell$ simultaneously); the rate of this event is $\alpha$. Similarly, a rod can exit from the lattice, with rate $\beta$, if it is occupying the site $i=L$ (and covering the remaining sites $i=L+1, L+2 \ldots L+\ell-1)$. A rod that occupies any site $i$ other than $i=L$ can hop forward, with step size unity (measured also in the unit of lattice spacing) and jump rate $p$, only if the site $i+\ell$ is empty.

$P(i, t)$ denotes the probability of finding a rod at site $i$ at time $t$.

We define $p(\underline{i} \mid i+\ell)$ as the conditional probability of finding site $i+\ell$ empty, if site $i$ is given to be occupied. It is straightforward to show that 37, 38.

$$
p(\underline{i} \mid i+\ell)=\frac{1-\rho \ell}{1+\rho-\rho \ell},
$$

where, $\rho$ is the occupational density of the system.

The master equations corresponding to the above explained dynamics are given below,

a: At site $i=1$,

$$
\frac{d P(i, t)}{d t}=\alpha P(\underbrace{0, \ldots, 0})-P(i, t) p(\underline{i} \mid i+\ell) p,
$$

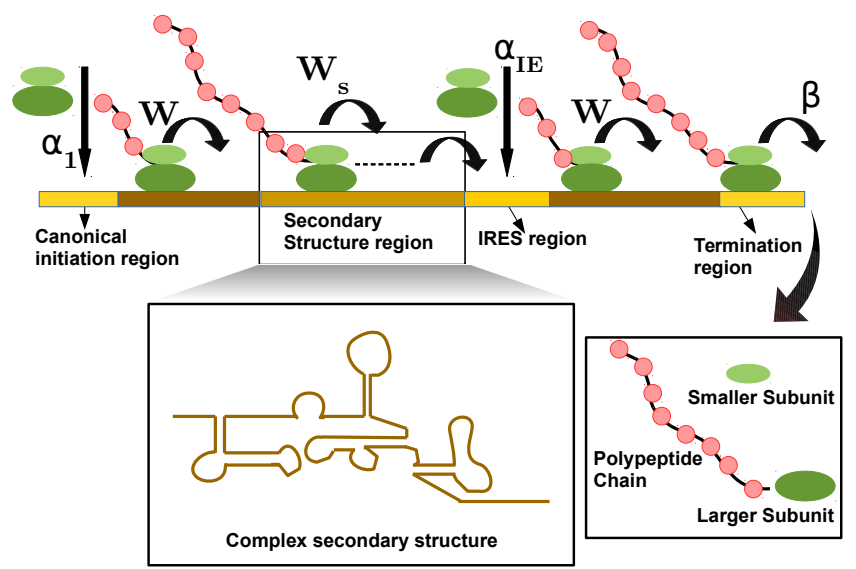

FIG. 1. (Color online) A cartoon depicting the phenomenon of unconventional translation initiated through IRES. The relative positions of the sites of canonical initiation and IRES with respect to that of the secondary structure of the mRNA track, along with the rates of the various kinetic processes, are shown schematically (see the text for details).

where, $P(\underbrace{0, \ldots, 0})$ is the probability that all sites from $i=1$ to $i=\ell$ are empty.

b: At site $i=L$,

$$
\frac{d P(i, t)}{d t}=P(i-1, t) p-P(i, t) \beta
$$

c: At all remaining sites,

$$
\begin{aligned}
\frac{d P(i, t)}{d t} & =P(i-1, t) p(\underline{i-1} \mid i+\ell-1) p \\
& -P(i, t) p(\underline{i} \mid i+\ell) p .
\end{aligned}
$$

Under steady state condition, flux $(J)$ in a uniform system is given by,

$$
J=p \frac{\rho(1-\rho \ell)}{(1+\rho-\rho \ell)}
$$

By taking, $\frac{d J}{d \rho}=0$, we get,

$$
\begin{gathered}
J_{M C}= \begin{cases}\frac{1}{(\sqrt{\ell}+1)^{2}} & \text { if } p=1, \\
\frac{p}{(\sqrt{\ell}+1)^{2}} & \text { if } p<1,\end{cases} \\
\rho_{c}^{M C}= \begin{cases}\frac{\sqrt{\ell}}{(\sqrt{\ell}+1)} & \text { if } p=1, \\
\frac{\sqrt{\ell}}{(\sqrt{\ell}+1)} & \text { if } p<1 .\end{cases}
\end{gathered}
$$

If initiation is the rate-limiting step (i.e., the rate $\alpha$ is the smallest of the three rates), then the system exhibits 


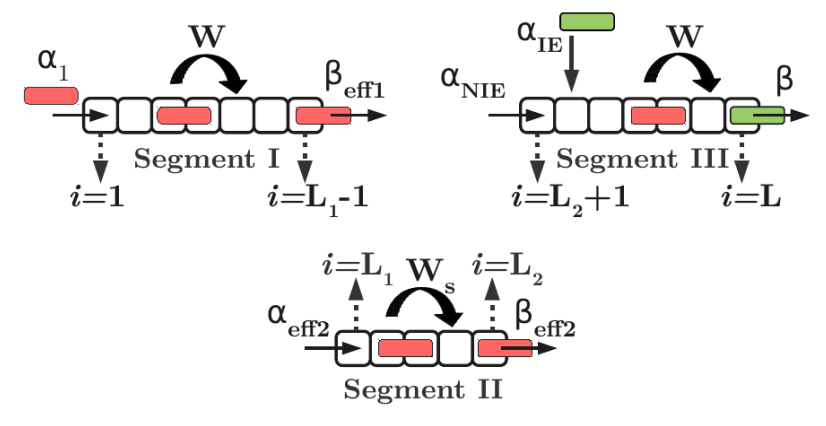

FIG. 2. (Color online) A schematic description of the twospecies exclusion model, intended to capture some of the key features of Fig 1 The whole lattice is divided into three segments. Segments I and III are regions where the rate of forward hopping of a rod is $W$, irrespective of the site and identity of the rod. The mid-segment II, that mimics the secondary structure of mRNA in Fig 1, is essentially an extended bottleneck because the forward jump rate $W_{s}$ of the rods in this segment is less than $W$. Sites $i=1$ and $i=i_{s}=L_{2}+n \ell+m$ are the sites of entry for the $\operatorname{rod}_{1}$ (with rate $\alpha_{1}$ in segment I) and for $\operatorname{rod}_{2}$ ( with rate $\alpha_{I E}$ in segment III), respectively. Thus, segments I and II are populated by only $\operatorname{rod}_{1}$ whereas a mixed population of $\operatorname{rod}_{1}$ and $\operatorname{rod}_{2}$ exists in segment III. $\alpha_{e f f 2}$ and $\alpha_{e f f 3}$ are effective rates of entry of the rods into the segments II and III, respectively; $\alpha_{e f f 3}$ gets contributions from $\alpha_{I E}$ as well as $\alpha_{N I E}$, the latter being the effective rate of entry of $\operatorname{rod}_{1}$ from segment II into segment III. Similarly, $\beta_{e f f 1}$ and $\beta_{e f f 2}$ are the effective rates of exit of the $\operatorname{rod}_{1}$ from segments I and II, respectively. For simplicity, we assume both species of rods to exit from the lattice, with the same rate $\beta$, from the same site $i=L$, irrespective of their identity. In this figure the rod length is taken as $\ell=2$ just for the purpose of illustration; in actual numerical calculations rod size has been taken as $\ell=10$ which captures the size of a ribosome more realistically.

\section{B. Brief introduction to Internal Ribosome Entry Site (IRES)}

The primary structure of a single DNA strand is a linear sequence of its monomeric subunits called nucleotides. The four species of nucleotides that can occur in this linear sequence are the analogs of four letters of a language in which nature encodes the genetic message. The same message is merely transcribed into the sequence of nucleotides on the messenger RNA (mRNA) molecule that is synthesized by a RNA polymerase (RNAP) machine using the DNA as a template. Triplets of nu- the low density (LD) phase. The steady state flux and the coverage density in the LD phase are given by

$$
\begin{gathered}
J_{L D}= \begin{cases}\frac{\alpha(1-\alpha)}{1+\alpha(\ell-1)} & \text { if } p=1, \\
\frac{\alpha(p-\alpha)}{p+\alpha(\ell-1)} & \text { if } p<1,\end{cases} \\
\rho_{c}^{L D}= \begin{cases}\frac{\alpha \ell}{1+\alpha(\ell-1)} & \text { if } p=1, \\
\frac{\alpha \ell}{p+\alpha(\ell-1)} & \text { if } p<1 .\end{cases}
\end{gathered}
$$

Similarly, if termination is the rate-limiting (i.e., slowest) step, the system would be in the high density (HD) phase. The steady state flux and the coverage density in the HD phase are given by

$$
\begin{aligned}
& J_{H D}= \begin{cases}\frac{\beta(1-\beta)}{1+\beta(\ell-1)} & \text { if } p=1, \\
\frac{\beta(p-\beta)}{p+\beta(\ell-1)} & \text { if } p<1,\end{cases} \\
& \rho_{c}^{H D}= \begin{cases}1-\beta & \text { if } p=1, \\
\frac{(p-\beta)}{p} & \text { if } p<1 .\end{cases}
\end{aligned}
$$

By comparing the fluxes (8 (a)), 9 (a)) and 10 (a)), we get the boundaries between different phases. 25] The main characteristics of the three dynamical phases, namely the low density (LD), high density (HD) and maximal current (MC) phases, that a TASEP for single species of hard rod exhibits on the $\alpha-\beta$ plane are summarized in Table (I] [8, 25, 26, 28, 35.

cleotides on a mRNA are called codons. Following the genetic code, each codon is translated into the corresponding amino acid, the monomeric subunits of a protein. Thus, the sequence of codons on a mRNA strand serves as the template for the synthesis of the corresponding protein thereby translating the genetic message from a language based on 4-letter alphabet to another language based on a 20-letter alphabet. The macromolecular machine that translates the genetic message is called a ribosome. From the perspective of statistical physics, a ribosome is a molecular motor 11, 93 that uses the mRNA template also as a track for its step-by-step for- 


\begin{tabular}{|c||c|c|c|}
\hline & Low Density (LD) & High Density (HD) & Maximal Current (MC) \\
\hline Phase boundary condition & $\alpha<\beta, \alpha<\frac{p}{1+\sqrt{\ell}}$ & $\beta<\alpha, \beta<\frac{p}{1+\sqrt{\ell}}$ & $\alpha>\frac{p}{1+\sqrt{\ell}, \beta>\frac{p}{1+\sqrt{\ell}}} \mid$ \\
Flux $(J)$ & $\frac{\alpha(p-\alpha)}{p+\alpha(\ell-1)}$ & $\frac{\beta(p-\beta)}{p+\beta(\ell-1)}$ & $\frac{p}{(\sqrt{\ell}+1)^{2}}$ \\
Coverage density $\left(\rho_{c}\right)$ & $\frac{\alpha \ell}{p+\alpha(\ell-1)}$ & $\frac{p-\beta}{(\sqrt{\ell}+1)}$
\end{tabular}

TABLE I. Comparison of phase boundary conditions, fluxes and coverage densities in three phases, namely the low density (LD), high density (HD) and maximal current (MC) phases.

ward movement. After translating each codon a ribosome steps forward to the next codon on the template mRNA; thus the step size of a ribosome motor on its track is identical to the linear size of a codon.

Often many ribosomes move simultaneously on the same mRNA track while each synthesizes a distinct copy of the same protein whose primary structure is encoded in the codon sequence on the template mRNA. Understanding the spatio-temporal organization of ribosomes in such traffic-like collective phenomenon in terms of a minimal mathematical model [9, 10, was the original motivation for formulation of TASEP [13, 14]. Since each ribosome simultaneously covers about 30 nucleotides (i.e., 10 codons) each was represented by a rod of length $\ell>1$, instead of a particle of length $\ell=1$, in the TASEP. The entry and exit of a rod in the TASEP capture the initiation and termination, respectively, of the process of translation by a ribosome. The hopping of a rod from one lattice site to the next mimics translation of successive codons on the mRNA template. Therefore, the average speed of a rod in the TASEP corresponds to the rate of elongation of a protein while the flux of the rods gives the overall rate of production of proteins.

Unlike DNA, which forms its iconic double-stranded helix, RNA can adopt wide varieties of secondary structures that are believed to have functionally important roles [99, 100]. Several of these secondary structures are, in fact, "signals" for alternative readout of the genetic message encoded in its own sequence. Such dynamic alteration of decoding, without any alteration of the genetic code, is called "recoding" 101, 102. Recoding phenomena are often referred to as "programmed" errors to distinguish these from random errors of decoding of genetic message. Except one special type of recoding that arises from programmed error at the level of transcription, almost all types of recoding take place via non-canonical translation and appear as programmed translational errors.

In canonical translation initiation takes place, effectively, at one end of the mRNA template. However, one of the non-canonical modes of translation [94 can get initiated from an "internal site" far downstream from the site of canonical initiation (see Fig 1). For obvious reason, this mode of non-canonical translation is named after the special site of initiation, namely, Internal Ribosome Entry Site (IRES) 98. The non-canonical routes of translation are widely exploited by viruses to hijack the ribosomal machineries of their host cells and to evade the anti-viral responses of the host [95, 96. In response to stress, cells are also believed to regulate translation via IRES route [97]. Therefore, a deep understanding of the interplay of canonical and non-canonical translation of a mRNA can help in developing strategies to combat viral infection.

The structural features of IRES and the circumstances which promote this mode of non-canonical initiation have been the main focus of investigation in the biology literature. One of the common features of IRES, observed across all known examples, is that IRES is located on, or just a few nucleotides downstrean from, a secondary structure formed by the mRNA template. However, to our knowledge, no attention has been paid so far on the consequences of IRES on ribosome traffic.

The main motivation for the two-species exclusion model developed in this paper is to explore possible effects of the interference of the flow of two species of ribosomes, engaged in canonical and non-canonical translation, on the overall nature of the ribosome traffic on a single mRNA. The phenomenon of non-canonical translation via IRES route has some similarities with transcriptional interference (TI) where two-species of RNAP motors, with their distinct sites of transcription initiation, interfere. Very recently a TASEP-based model of TI has been reported elsewhere 103. However, as we shall demonstrate in this paper, the secondary structure of the mRNA template immediately upstream from the IRES, serves as an additional regulator of the extent of interference of the flow of the two species of ribosomes. Consequently, much richer varieties of spatio-temporal organization of the ribosomes, under different levels of stability of the mRNA secondary structure, are expected in the model developed here than those reported in the 
context of TI in ref. [103.

\section{Two-species TASEP with hard rods inspired by IRES}

The schematic diagram of the model is shown in Fig. (2). This model consists of a one dimensional lattice of size $L+\ell-1$ and multiple identical rods of two different species. Size of a rod is $\ell$ in the units of lattice sites (i.e. it covers $\ell$ sites simultaneously) and it is assumed to be identical for both species 1 and 2. Here, we denote the position of a rod (either rod of species 1 or 2) by the lattice site at which the left most edge of the rod is located. If the left most edge of the rod is at site $i$, then, site $i$ is known as 'occupied' site and all remaining sites $i+1, i+2, \ldots, i+\ell-1$ are 'covered' sites. In this model, the whole lattice is divided into three segments: segment I consists of site $i=1$ to site $i=L_{1}-1$ (i.e. $1 \leq i \leq L_{1}-1$ ), segment II from site $i=L_{1}$ to site $i=L_{2}$ (i.e. $L_{1} \leq i \leq L_{2}$ ) and segment III from site $i=L_{2}+1$ to site $i=L+\ell-1$ (i.e. $L_{2}+1 \leq i \leq L+\ell-1$ ).

A new rod can enter via two possible pathways: a) through canonical initiation and b) through noncanonical initiation. In the "canonical initiation" pathway a new rod can attach to the first lattice site (i.e. at $i=1$ ) with rate $\alpha_{1}$, if all the $\ell$ sites $1,2, \ldots \ell$ are simultaneously empty (i.e. neither occupied nor covered). In the alternative "non-canonical initiation" pathway a new rod can attach to a special internal entry site (i.e. at $i=i_{s}=L_{2}+n \ell+m$, where $n, m$ are two integers), with rate $\alpha_{I E}$, if all sites from $i_{s}$ to $i_{s}+\ell-1$ are neither occupied nor covered simultaneously, and it will be referred to as "non-canonical initiation". Based on the entry pathway used, the rods can be divided into two groups, a) those which enter through the site $i=1$ will be referred to as of type $\operatorname{rod}_{1}$, and b) those which enter through the site $i=i_{s}$ will be referred to as of type $\operatorname{rod}_{2}$.

In contrast to distinct sites of entry for the two species $\operatorname{rod}_{1}$ and $\operatorname{rod}_{2}$, there is only a single downstream site on the lattice from where both types of rods exit. If a rod is occupying site $i=L$ (and is covering the sites $i=L+1, L+2, \ldots, L+\ell-1)$, it can detach from the lattice with rate $\beta$, irrespective of whether it is $\operatorname{rod}_{1}$ or $\operatorname{rod}_{2}$.

Except for initiation and termination, throughout the lattice, a rod can jump only in the forward direction, with step size of unity measured in the units of lattice spacing. The rate of forward jump is same at all sites in the segments I and III; this rate is $W$ irrespective of whether the rod is $\operatorname{rod}_{1}$ or $\operatorname{rod}_{2}$. Jump rate $W_{s}$ inside segment II is less than $W$ and is assumed to depends on the stability of the pseudoknot according to the following expression

$$
W_{s} / W=\gamma=\exp (-a \widehat{\Delta G}),
$$

where, $\widehat{\Delta G}$ is proportional to the free energy barrier to be overcome by a rod to proceed forward by one step and $a$ is a constant of proportionality that is intended to capture the complexities of the pseudoknot structure. For all numerical calculations reported in this paper the numerical values $W=1$ and $a=3$ has been chosen. Thus, the kinetics of the system is governed by the following prescription:

Inside segment I,

a: A new $\operatorname{rod}\left(\operatorname{rod}_{1}\right)$ can attach at site $i=1$ with rate $\alpha_{1}$, if all sites from $i=1$ to $i=\ell$ are neither covered nor occupied, simultaneously.

b: At any site, a rod $\left(\operatorname{rod}_{1}\right)$ can move forward with step size 1 and rate $W$, if it is occupying site $i$ and the target site $i+\ell$ is not occupied.

c: $\mathrm{A} \operatorname{rod}\left(\operatorname{rod}_{1}\right)$ can exit from segment I with rate $W$, if it is at site $i=L_{1}-1$ and the target site $i+\ell$ in segment II is not occupied. The exclusion process on the segment I can be regarded as a single TASEP for $\operatorname{rod}_{1}$ with the effective exit rate $\beta_{\text {eff } 1}$ for $\operatorname{rod}_{1}$ from segment I.

Inside segment II,

a: The right edge of a rod $\left(\operatorname{rod}_{1}\right)$ can enter in segment II with rate $W$, if its left edge is at site $i=L_{1}-\ell$ and the target site $i+\ell$ is not occupied. The exclusion process in segment II can be regarded as a single TASEP for $\operatorname{rod}_{1}$ with the effective rate $\alpha_{e f f 2}$ for the entry of $\operatorname{rod}_{1}$ into the segment II.

b: At any site a rod $\left(\operatorname{rod}_{1}\right)$ can move forward with step size 1 and rate $W_{s}$, if it is occupying site $i$ and the target site $i+\ell$ is not occupied.

c: A $\operatorname{rod}\left(\operatorname{rod}_{1}\right)$ can exit from segment II with rate $W_{s}$, if its left edge is at site $i=L_{2}$ and the target site $i+\ell$ in segment III is not occupied by any other rod. Thus the exclusion process in segment II can be regarded as a TASEP with the effective rate $\beta_{e f f 2}$ of exit of $\operatorname{rod}_{1}$ from segment II.

Inside segment III,

a: A $\operatorname{rod}_{1}$ can enter segment III from segment II with rate $W_{s}$, if its left edge is at site $i=L_{2}-\ell+1$ and the target site $i+\ell$ is not occupied by any other rod. Thus, the effective rate of entry of $\operatorname{rod}_{1}$ into segment III is $\alpha_{N I E}$.

b: Inside this segment there is one special site at $i=i_{s}$ to which a new $\operatorname{rod}_{2}$ can attach with rate $\alpha_{I E}$ if none of the sites from $i_{s}$ to $i_{s}+\ell-1$ are covered. Thus, the exclusion process in segment III can be regarded as a TASEP of mixed population of $\operatorname{rod}_{1}$ and $\operatorname{rod}_{2}$ with the total entry rate $\alpha_{\text {eff } 3 \text {. }}$

c: A rod $\left(\operatorname{rod}_{1}\right.$ or $\left.\operatorname{rod}_{2}\right)$, can completely detach from the lattice with rate $\beta$, if it is occupying site $i=L$ and covering remaining sites $i=L+1, \ldots, L+\ell-1$. The rate of detachment is assumed to be identical for both $\operatorname{rod}_{1}$ and $\operatorname{rod}_{2}$.

d: At all other sites, a rod $\left(\operatorname{rod}_{1}\right.$ or $\left.\operatorname{rod}_{2}\right)$ can move forward with step size 1 and rate $W$, if its left edge is located at site $i$ and the target site $i+\ell$ is not occupied by any other rod.

The effective rate constants and their defining expressions are given in table II. Note that the exclusion process 


\begin{tabular}{|c|c|}
\hline Rate constant & Expression \\
\hline \hline$\alpha_{e f f 2}$ & $\alpha_{e f f 2}=W \rho_{c 1} / \ell$ \\
\hline$\alpha_{N I E}$ & $\alpha_{N I E}=W_{s} \rho_{c 2} / \ell$ \\
\hline$\alpha_{e f f 3}$ & $\alpha_{e f f 3}=\alpha_{N I E}+\alpha_{I E}$ \\
\hline$\beta_{e f f 1}$ & $\beta_{e f f 1}=W P_{1}\left(\underline{L_{1}-1} \mid L_{1}+\ell-1\right)$ \\
\hline$\beta_{e f f 2}$ & $\beta_{e f f 2}=W_{s} P_{1}\left(\underline{L_{2}} \mid L_{2}+\ell\right)$ \\
\hline
\end{tabular}

TABLE II. The effective rate constants and their defining expressions.

in each of the segments I and II are single-species exclusion process whereas that in segment III is a two-species exclusion process. However, for simplicity, we assume the same hopping rate $W$ and exit rate $\beta$ in this segment for both species of the rods.

\section{Two models of Non-canonical initiation}

\section{- Signal Independent Initiation:}

In the first model, one assumes that no correlation exists between the canonical and non-canonical initiation so that the rates $\alpha_{1}$ and $\alpha_{I E}$ of the entry of $\operatorname{rod}_{1}$ and $\operatorname{rod}_{2}$ in the segments I and III, respectively are two independent parameters.

- Signal Dependent Initiation:

In the alternative model, the rates $\alpha_{1}$ and $\alpha_{I E}$ of canonical and non-canonical initiation, respectively, cannot be treated as independent parameters. Instead, the rates of entry of $\operatorname{rod}_{1}$ and $\operatorname{rod}_{2}$ are controlled by an external signal according to the prescription

$$
\alpha_{1}=s \alpha,
$$

and

$$
\alpha_{I E}=(1-s) \alpha .
$$

where, $\alpha$ is the total rate of initiation and an external signal decides the fraction, denoted by ' $s$, of initiation events that occur via the canonical route. We compare the results for these two models in the concluding section.

\section{E. Quantities of interest}

Let $P_{\mu}(i, t)$ denote the probability of finding a rod of type $\mu(\mu=1,2)$ at site $i$ at time $t$. We use the symbols $J_{\text {seg } 1}, J_{\text {seg } 2}$ and $J_{\text {seg } 3}$ to denote the net flux of the rods in the segments I, II and III, respectively. Under steady state conditions, $J_{\text {seg } \mu}(\mu=1,2,3)$ would be given by either $(9)$ (a) or 110 (a) or 8 (a) depending on whether the segment under consideration is in the $\mathrm{LD}$, or $\mathrm{HD}$ or $\mathrm{MC}$ phase; the entry and exit rates to be used in these equations are the effective rates for the respective segments, namely $\alpha_{1}, \beta_{e f f 1}$ for I, $\alpha_{e f f 2}, \beta_{e f f 2}$ for II and $\alpha_{e f f 3}, \beta$ for III. Under steady state condition, flux in the three segments must satisfy the following conditions:

$$
J_{\text {seg } 1}=J_{\text {seg } 2}
$$

and

$$
J_{\text {seg } 3}=J_{\text {seg } 1}+\alpha_{I E} P(\underbrace{0, \ldots, 0}_{\ell}) .
$$

where $P(\underbrace{0, \ldots, 0}_{\ell})$ is the probability that none of the sites from $i=i_{s}$ to $i=i_{s}+\ell-1$ are covered.

The net flux gets contributions from the forward movemetns of both types of rods. The fluxes $J_{1}$ and $J_{2}$ of $\operatorname{rod}_{1}$ and $\operatorname{rod}_{2}$, respectively, which are the number of RNA molecules synthesized per unit time in the canonical and non-canonical translation processes, are defined by

$$
J_{\mu}=P_{\mu}(L, t) \beta \quad(\mu=1,2) .
$$

Since, the system consists of three segments where each has some distinct kinetics of the rods, the coverage densities in these three segments can be different from each other. We use the symbols $\rho_{c 1}, \rho_{c 2}$ and $\rho_{c 3}$ for the coverage densities in segments I, II and III, respectively. The overall spatio-temporal organization of the rods, arising from the inhomogeneous density profiles, but uniform flux, in the three-segment system, can be studied by displaying all possible composite phases on the phase diagram. Each composite phase comprises of the three phases that can exist in three segments. In principle, each segment can exist in one of the three possible phases i.e. low density (LD), high density (HD) and maximal current $(\mathrm{MC})$ phase. The composite phase diagrams will be denoted by the symbols $X / Y / Z$, where $X, Y, Z$ correspond to the phases in the segments I, II, II, respectively, and where each of the three symbols $X, Y, Z$ can be either LD or HD or MC.

\section{F. Master equations}

We define $p(\underline{i} \mid i+\ell)$ as the conditional probability of finding site $i+\ell$ empty, if site $i$ is given to be occupied. It is straightforward to show that [37, 38]

$$
p(\underline{i} \mid i+\ell)=\frac{1-\rho \ell}{1+\rho-\rho \ell},
$$

where, $\rho$ is the occupational density (i.e., number density) of the rods in the system. Similarly, we define $P(\underbrace{0, \ldots, 0}_{\ell, 0})$ as the probability that all sites from $i=i_{s}$ $\underbrace{}_{\ell}$

to $i=i_{s}+\ell-1$ are neither occupied nor covered, simultaneously. Expression of $P(\underbrace{0, \ldots, 0})$ in terms of occupational density $\rho_{3}$ of III (when $i_{s}=L_{2}+n \ell+m$ 
and $n \geq 1$ ), is given by (see the appendix for full derivation of (Eq. A3, A5 and A6),

$$
P(\underbrace{0, \ldots, 0}_{\ell})=\frac{2\left(1-\rho_{3}\right)}{2\left(1-\rho_{3}\right)\left(1+\rho_{3}^{\prime}\right)+\rho_{3} \ell\left(2+\rho_{3}^{\prime}\right)} .
$$

where, $\rho_{3}^{\prime}=\rho_{3}(\ell-1)$.

Master equations, under mean field approximation (MFA), corresponding to the stochastic kinetics explained above are given by the following expressions:

(A) For $\operatorname{rod}_{1}$ in segment $\mathrm{I}$,

a: At site $i=1$,

$$
\frac{d P_{1}(i, t)}{d t}=\alpha_{1}\left[1-\sum_{k=1}^{\ell} P_{1}(k, t)\right]-P_{1}(i, t) p(\underline{i} \mid i+\ell) W
$$

b: At all remaining sites,

$$
\begin{aligned}
\frac{d P_{1}(i, t)}{d t} & =P_{1}(i-1, t) p(\underline{i-1} \mid i+\ell-1) W \\
& -P_{1}(i, t) p(\underline{i} \mid i+\ell) W
\end{aligned}
$$

In segment $\mathrm{II}$,

$$
\begin{aligned}
\frac{d P_{1}(i, t)}{d t} & =P_{1}(i-1, t) p(\underline{i-1} \mid i+\ell-1) W_{s} \\
& -P_{1}(i, t) p(\underline{i} \mid i+\ell) W_{s},
\end{aligned}
$$

In segment III,

a: At site $i=L$,

$$
\frac{d P_{1}(i, t)}{d t}=P_{1}(i-1, t) W-P_{1}(i, t) \beta,
$$

b: At all remaining sites,

$$
\begin{aligned}
\frac{d P_{1}(i, t)}{d t} & =P_{1}(i-1, t) p(\underline{i-1} \mid i+\ell-1) W \\
& -P_{1}(i, t) p(\underline{i} \mid i+\ell) W .
\end{aligned}
$$

(B) For $\operatorname{rod}_{2}$

if $i<i_{s}$,

$$
P_{2}(i, t)=0
$$

in segment III

$$
\begin{aligned}
& \text { a: At site } i=i_{s}, \\
& \qquad \begin{aligned}
\frac{d P_{2}(i, t)}{d t} & =\alpha_{I E} P(\underbrace{0, \ldots, 0}_{\ell}) \\
& -P_{2}(i, t) p(\underline{i} \mid i+\ell) W,
\end{aligned}
\end{aligned}
$$

b: At site $i=L$,

$$
\frac{d P_{2}(i, t)}{d t}=P_{2}(i-1, t) W-P_{2}(i, t) \beta,
$$

c: At all remaining sites,

$$
\begin{aligned}
\frac{d P_{2}(i, t)}{d t} & =P_{2}(i-1, t) p(\underline{i-1} \mid i+\ell-1) W \\
& -P_{2}(i, t) p(\underline{i} \mid i+\ell) W
\end{aligned}
$$

\section{RESULTS}

We have followed two different approaches for studying the combined effect of initiation rates $\alpha_{1}, \alpha_{I E}$ and the jump rate $W_{s}$ in segment II (or, more precisely, the ratio $\gamma=W_{s} / W$ ) on the spatio-temporal organization of the two species of rods in the system. Our first approach is based on an analysis of the master equations formulated above under MFA. This analysis is primarily analytical derivation of the phase boundaries and fluxes in the steady states of the system.

In our second approach, we carry out extensive MonteCarlo (MC) simulations of the same model. In the MC simulations, starting from an arbitrary initial state, the state of the system was updated for sufficiently large number of MC steps to achieve steady state after which the relevant data were collected over the next $1 \times 10^{5} \mathrm{MC}$ steps. In order to convert the rates (with units $\mathrm{s}^{-1}$ ) of the various processes into the corresponding dimensionless probabilities we have used the prescription

$$
p_{k}=k d t
$$

where, $p_{k}$ is the probability corresponding to an arbitrary rate constant $k$ and $d t$ is a small time step; for all the numerical data presented graphically in this paper we have chosen $d t=0.01 \mathrm{~s}$.

\section{A. Analytical derivations of phase boundaries and fluxes}

We can model our system as a combination of three TASEP, where a TASEP on the left (right) of a given segment acts, effectively, as a reservoir from where rods enter (to which rods exit) the given segment. Therefore, at first sight, it may appear that the entire system could be found in $3^{3}=27$ possible phases. But, because of some symmetry requirements and steady state conditions not all 27 phases can exist in the system.

The steady-state flux inside segments I and II should always be identical. If MC phase exist in segment I, the flux inside this segment should be equal to, $J_{\text {seg } 1}=1 /(\sqrt{\ell}+1)^{2}$. However, if segment II is in MC phase, $J_{\text {seg } 2}=W_{s} /(\sqrt{\ell}+1)^{2}<1 /(\sqrt{\ell}+1)^{2}$, when $W_{s}<1$. Therefore, those composite phases are ruled out, where the segment I is in MC phase while the 
segment II is not. Similarly, if both the segments I and II are in HD phase, the probability of finding segment III in LD phase is approaching towards zero value, when $\alpha_{I E} \neq 0$. Taking into account all the conditions arising from symmetry considerations, the model developed in this paper can exist in only seven possible phase. We characterize here only those seven phases based on analytical treatment as well as MC simulations.

We now begin presentation of our results by analytically deriving the phase boundaries of the system.

\section{Phase 1: LD/LD/LD phase}

Since all the segments are in LD phases, this composite phase of the system is specified by the following conditions,

$$
\begin{gathered}
\alpha_{1}<\frac{1}{\sqrt{\ell}+1}, \quad \alpha_{1}<\beta_{e f f 1}, \\
\alpha_{e f f 2}<\frac{W_{s}}{\sqrt{\ell}+1}, \quad \alpha_{e f f 2}<\beta_{e f f 2}, \\
\alpha_{e f f 3}<\frac{1}{\sqrt{\ell}+1}, \quad \alpha_{e f f 3}<\beta .
\end{gathered}
$$

Under steady state condition the fluxes of the rods that follow from the equations $(9)(a)$, are given by

$$
\begin{aligned}
& J_{\text {seg } 1}=\frac{\alpha_{1}\left(1-\alpha_{1}\right)}{1+\alpha_{1}(\ell-1)}, \quad(a) \\
& J_{\text {seg } 2}=\frac{\alpha_{e f f 2}\left(W_{s}-\alpha_{e f f 2}\right)}{W_{s}+\alpha_{e f f 2}(\ell-1)}, \quad(b) \\
& J_{\text {seg } 3}=\frac{\alpha_{e f f 3}\left(1-\alpha_{e f f 3}\right)}{1+\alpha_{e f f 3}(\ell-1)}, \quad(c)
\end{aligned}
$$

while, from the equations $(9)$ (b), the corresponding coverage densities are given by

$$
\begin{aligned}
\rho_{c 1} & =\frac{\alpha_{1} \ell}{1+\alpha_{1}(\ell-1)}, \quad(a) \\
\rho_{c 2} & =\frac{\alpha_{e f f 2} \ell}{W_{s}+\alpha_{e f f 2}(\ell-1)}, \\
\rho_{c 3} & =\frac{\alpha_{e f f 3} \ell}{1+\alpha_{e f f 3}(\ell-1)} . \quad(c)
\end{aligned}
$$

Now imposing the condition (14), i.e., equating $[30$ (a) and 30 (b), we get

$$
\frac{\alpha_{1}\left(1-\alpha_{1}\right)}{1+\alpha_{1}(\ell-1)}-\frac{\alpha_{e f f 2}\left(W_{s}-\alpha_{e f f 2}\right)}{W_{s}+\alpha_{e f f 2}(\ell-1)}=0
$$

solving which for the unknown $\alpha_{e f f 2}$ we get,

$$
\alpha_{e f f 2}=\frac{-t_{1}-\sqrt{t_{1}^{2}-4 t_{2}}}{2 t_{3}},
$$

where,

$$
\begin{gathered}
t_{1}=-\left(\alpha_{1}^{2} \ell-\alpha_{1}^{2}+\alpha_{1} \ell W_{s}-\alpha_{1} \ell-\alpha_{1} W_{s}+\alpha_{1}+W_{s}\right), \\
t_{2}=\left(\alpha_{1} \ell-\alpha_{1}+1\right)\left(\alpha_{1} W_{s}-\alpha_{1}^{2} W_{s}\right) \\
t_{3}=\alpha_{1} \ell-\alpha_{1}+1
\end{gathered}
$$

Note that of the two solutions of the quadratic equation we have chosen the one with the negative sign to be the physical solution because $\alpha_{\text {eff } 2}$ should be less than $W_{s} /(\sqrt{\ell}+1)$.

Combining Eqn. (33) and the condition (29), we get the maximum physically admissible value of the $\alpha_{1}$ upto which both the segments I and II would exist in LD phases. Thus, substituting Eq. (33) into (29) we get

$$
\frac{-t_{1}-\sqrt{t_{1}^{2}-4 t_{2}}}{2 t_{3}}<\frac{W_{s}}{(\sqrt{\ell}+1)},
$$

which, for example, for $\ell=10$ reduces to the condition

$$
\alpha_{1}<\frac{t_{11}-t_{12}}{18},
$$

for the occurrence of LD phase in both the segments I and II where

$$
\begin{gathered}
t_{11}=2 \sqrt{10} W_{s}-11 W_{s}+9 \\
t_{12}=\sqrt{\left(2 \sqrt{10} W_{s}-11 W_{s}+9\right)^{2}-(44-8 \sqrt{10}) W_{s}} .
\end{gathered}
$$

Substituting the expression (33) for $\alpha_{\text {eff } 2}$ into those of $J_{\text {seg2 } 2}$ in (30) and $\rho_{c 2}$ in (31) we get the flux $J_{\text {seg } 2}$ and coverage density $\rho_{c 2}$ in terms of $\alpha_{1}$ and $W_{s}$,

$$
\rho_{c 2}=\frac{\ell\left(-t_{1}-\sqrt{t_{1}^{2}-4 t_{2}}\right)}{2 t_{3}\left(W_{s}+\frac{(\ell-1)\left(-t_{1}-\sqrt{t_{1}^{2}-4 t_{2}}\right)}{2 t_{3}}\right)} .
$$

Furthermore, substituting the expressions thus obtained for $\alpha_{e f f 2}$ and $\rho_{c 2}$ into

$$
\alpha_{N I E}=W_{s} \frac{\rho_{c 2}}{\ell},
$$

we get the expression

$$
\alpha_{N I E}=\frac{W_{s}\left(-t_{1}-\sqrt{t_{1}^{2}-4 t_{2}}\right)}{2 t_{3}\left(W_{s}+\frac{(\ell-1)\left(-t_{1}-\sqrt{t_{1}^{2}-4 t_{2}}\right)}{2 t_{3}}\right)},
$$

for $\alpha_{N I E}$, and hence

$$
\begin{aligned}
\alpha_{e f f 3} & =\alpha_{I E}+\alpha_{N I E} \\
& =\alpha_{I E}+\frac{W_{s}\left(-t_{1}-\sqrt{t_{1}^{2}-4 t_{2}}\right)}{2 t_{3}\left(W_{s}+\frac{(\ell-1)\left(-t_{1}-\sqrt{t_{1}^{2}-4 t_{2}}\right)}{2 t_{3}}\right)} .
\end{aligned}
$$


From condition 29],

$$
\begin{aligned}
\beta>\alpha_{\text {eff } 3} & =\alpha_{I E} \\
& +\frac{W_{s}\left(-t_{1}-\sqrt{t_{1}^{2}-4 t_{2}}\right)}{2 t_{3}\left(W_{s}+\frac{(\ell-1)\left(-t_{1}-\sqrt{t_{1}^{2}-4 t_{2}}\right)}{2 t_{3}}\right)} .
\end{aligned}
$$

The canonical and non-canonical fluxes in the $\mathrm{LD} / \mathrm{LD} / \mathrm{LD}$ phase are given by the expressions

$$
J_{1}=J_{s e g 1}=J_{s e g 2}=\frac{\alpha_{e f f 2}\left(W_{s}-\alpha_{e f f 2}\right)}{\left(W_{s}+\alpha_{e f f 2}(\ell-1)\right)},
$$

and

$$
J_{2}=\frac{\alpha_{e f f 3}\left(1-\alpha_{e f f 3}\right)}{\left(1+\alpha_{e f f 3}(\ell-1)\right)}-\frac{\alpha_{e f f 2}\left(W_{s}-\alpha_{e f f 2}\right)}{\left(W_{s}+\alpha_{e f f 2}(\ell-1)\right)}
$$

Phase 2: LD/LD/HD phase The LD/LD/HD phase is specified by the following conditions,

$$
\begin{aligned}
\alpha_{1} & <\frac{1}{\sqrt{\ell}+1}, & \alpha_{1}<\beta_{e f f 1}, \\
\alpha_{e f f 2} & <\frac{W_{s}}{\sqrt{\ell}+1}, & \alpha_{e f f 2}<\beta_{e f f 2}, \\
\beta & <\frac{1}{\sqrt{\ell}+1}, & \beta<\alpha_{e f f 3},
\end{aligned}
$$

in terms of the effective rates; the corresponding steadystate fluxes that follow from the equations (9)(a) and 10 (a), would be

$$
\begin{aligned}
& J_{\text {seg } 1}=\frac{\alpha_{1}\left(1-\alpha_{1}\right)}{1+\alpha_{1}(\ell-1)}, \quad(a) \\
& J_{\text {seg } 2}=\frac{\alpha_{e f f 2}\left(W_{s}-\alpha_{e f f 2}\right)}{W_{s}+\alpha_{e f f 2}(\ell-1)}, \\
& J_{\text {seg } 3}=\frac{\beta(1-\beta)}{1+\beta(\ell-1)}, \quad(c)
\end{aligned}
$$

while, from the equations 99 (b) and 10 (b) the corresponding coverage densities would be given by

$$
\begin{aligned}
\rho_{c 1} & =\frac{\alpha_{1} \ell}{1+\alpha_{1}(\ell-1)}, \quad(a) \\
\rho_{c 2} & =\frac{\alpha_{e f f 2} \ell}{W_{s}+\alpha_{e f f 2}(\ell-1)}, \\
\rho_{c 3} & =1-\beta . \quad(c)
\end{aligned}
$$

Now imposing the condition (14), i.e., equating (49) (a) and 49p(b), and solving for the unknown $\alpha_{e f f 2}$ we get the same expression (33). This, however, is not surprising because $\alpha_{\text {eff } 2}$ for both the phases 1 and 2 is a characteristics of the $\mathrm{LD} / \mathrm{LD}$ interface.

Next imposing Eq. 15, i.e., equating the fluxes of segment II and III we get,

$$
\frac{\beta(1-\beta)}{1+\beta(\ell-1)}=\frac{\alpha_{e f f 2}\left(W_{s}-\alpha_{e f f 2}\right)}{W_{s}+\alpha_{e f f 2}(\ell-1)}+\alpha_{I E} P(\underbrace{0, \ldots, 0}_{\ell}) .
$$

Substituting the expression

$$
P(\underbrace{0, \ldots, 0}_{\ell})=\frac{2 \beta}{2 \beta\left(1+\beta^{\prime}\right)+(1-\beta) \ell\left(2+\beta^{\prime}\right)} .
$$

(see the derivation of Eq. $\mathrm{A9}$ in the Appendix for the $\mathrm{LD} / \mathrm{LD} / \mathrm{HD}$ phase), where, $\beta^{\prime}=(1-\beta)(\ell-1)$, into Eq.51) we get

$$
\begin{gathered}
\frac{\beta(1-\beta)}{1+\beta(\ell-1)}=\frac{\alpha_{e f f 2}\left(W_{s}-\alpha_{e f f 2}\right)}{W_{s}+\alpha_{e f f 2}(\ell-1)} \\
+\frac{2 \alpha_{I E} \beta}{2 \beta\left(1+\beta^{\prime}\right)+(1-\beta) \ell\left(2+\beta^{\prime}\right)} .
\end{gathered}
$$

Solving this quartic equation for $\beta$, we get four solutions of which two are negative solutions and, hence, inadmissible. Out of two positive solutions we have chosen the one with the negative sign to be the physical solution because, as dictated by (48), $\beta$ must be less than $1 /(\sqrt{\ell}+1)$. Since the general expression for $\beta$ is too long to be reproduced here, the expression of $\beta$ for the special values of the parameter set: $W_{s}=0.9, \ell=10, \alpha_{I E}=0.15$ is given in the appendix $B$.

Substituting the expression (33) for $\alpha_{\text {eff } 2}$ in the solution for $\beta$ thus obtained, we get the relation between $\alpha_{1}$ and $\beta$ in terms of $W_{s}, \ell$ and $\alpha_{I E}$. This relation gives us a transition line which separates the $\mathrm{LD} / \mathrm{LD} / \mathrm{HD}$ from HD/HD/HD phase. During this transition, density of segment I and II changes discontinuously. Thus, the conditions (45) and (48), written in terms of the effective rates, now reduce to

$$
\begin{aligned}
\alpha_{I E} & +\frac{W_{s}\left(-t_{1}-\sqrt{t_{1}^{2}-4 t_{2}}\right)}{2 t_{3}\left(W_{s}+\frac{(\ell-1)\left(-t_{1}-\sqrt{t_{1}^{2}-4 t_{2}}\right)}{2 t_{3}}\right)}>\beta>\left[-s_{0}\right. \\
& -0.5 \sqrt{s_{0}-s_{1}-\left(s_{2} / s_{3}\right)-s_{4}} \\
& \left.-0.5 \sqrt{s_{5}-s_{6}+\left(s_{2} / s_{3}\right)+s_{4}-\left(s_{7} / s_{8}\right)}\right]
\end{aligned}
$$

for the composite phase $\mathrm{LD} / \mathrm{LD} / \mathrm{HD}$ of the system where $s_{\mu}(\mu=0,1, \cdots 8)$ are expressed in terms of the basic rate constants of the model (see appendix $\mathrm{B}$ for an example).

Finally, in the LD/LD/HD phase the canonical and non-canonical fluxes are given by

$$
J_{1}=J_{s e g 1}=J_{s e g 2}=\frac{\alpha_{e f f 2}\left(W_{s}-\alpha_{e f f 2}\right)}{\left(W_{s}+\alpha_{e f f 2}(\ell-1)\right)},
$$

and

$$
J_{2}=\frac{\beta(1-\beta)}{(1+\beta(\ell-1))}-\frac{\alpha_{e f f 2}\left(W_{s}-\alpha_{e f f 2}\right)}{\left(W_{s}+\alpha_{e f f 2}(\ell-1)\right)} .
$$

respectively, where $\alpha_{\text {eff } 2}$ is given by (33).

Phase 3: LD/LD/MC phase

The $\mathrm{LD} / \mathrm{LD} / \mathrm{MC}$ phase is specified by the conditions, 


$$
\begin{array}{rlrl}
\alpha_{1} & <\frac{1}{\sqrt{\ell}+1}, & \alpha_{1}<\beta_{\text {eff } 1,} \\
\alpha_{e f f 2} & <\frac{W_{s}}{\sqrt{\ell}+1}, & & \alpha_{e f f 2}<\beta_{\text {eff } 2}, \\
\beta & >\frac{1}{\sqrt{\ell}+1}, & \alpha_{e f f 3}>\frac{1}{\sqrt{\ell}+1},
\end{array}
$$

in terms of the effective rates; the corresponding steadystate fluxes that follow from the equations 99 (a) and (8) (a), would be

$$
\begin{aligned}
J_{s e g 1} & =\frac{\alpha_{1}\left(1-\alpha_{1}\right)}{1+\alpha_{1}(\ell-1)}, \quad(a) \\
J_{s e g 2} & =\frac{\alpha_{e f f 2}\left(W_{s}-\alpha_{e f f 2}\right)}{W_{s}+\alpha_{e f f 2}(\ell-1)} \\
J_{\text {seg } 3} & =\frac{1}{(\sqrt{\ell}+1)^{2}}, \quad(c)
\end{aligned}
$$

while, from the equations $\sqrt{9}(\mathrm{~b})$ and $\sqrt{8}(\mathrm{~b})$ the corresponding coverage densities would be given by

$$
\begin{aligned}
\rho_{c 1} & =\frac{\alpha_{1} \ell}{1+\alpha_{1}(\ell-1)}, \quad(a) \\
\rho_{c 2} & =\frac{\alpha_{e f f 2} \ell}{W_{s}+\alpha_{e f f 2}(\ell-1)}, \\
\rho_{c 3} & =\frac{\sqrt{\ell}}{\sqrt{\ell}+1} . \quad(c)
\end{aligned}
$$

Imposition of the condition (14), as before, yields the ex-

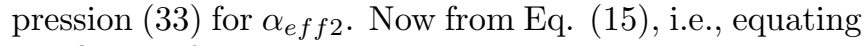
the fluxes of segment II and IIIi, we get

$$
\frac{1}{(\sqrt{\ell}+1)^{2}}=\frac{\alpha_{e f f 2}\left(W_{s}-\alpha_{e f f 2}\right)}{W_{s}+\alpha_{e f f 2}(\ell-1)}+\alpha_{I E} P(\underbrace{0, \ldots, 0}_{\ell}),
$$

substitution of

$$
P(\underbrace{0, \ldots, 0}_{\ell})=\frac{4}{2(\ell+1)+\ell(\ell+3)},
$$

for $\mathrm{LD} / \mathrm{LD} / \mathrm{MC}$ phase (see the derivation of Eq. A11 in the Appendix) into Eq. 59. leads to

$$
\begin{aligned}
\frac{1}{(\sqrt{\ell}+1)^{2}} & =\frac{\alpha_{e f f 2}\left(W_{s}-\alpha_{e f f 2}\right)}{W_{s}+\alpha_{e f f 2}(\ell-1)} \\
& +\frac{4 \alpha_{I E}}{2(\ell+1)+\ell(\ell+3)} .
\end{aligned}
$$

Solving this quadratic equation wa we two solutions for of $\alpha_{e f f 2}$; we have chosen the solution with the negative sign to be the physical solution because, as the eq. 56 requires, $\alpha_{\text {eff } 2}$ must be less than $W_{s} /(\sqrt{\ell}+1)$. The Eq.59 leads to

$$
\alpha_{e f f 2}=\frac{-t_{4}-\sqrt{t_{4}^{2}-4 t_{5} t_{6}}}{2 t_{6}}
$$

The general expresions for $t_{4}, t_{5}$ and $t_{6}$ are given in the appendix C(see Eq. C2, C3 and C4).

The expression 62 of $\alpha_{\text {eff2 }}$, that we get here in terms of $W_{s}, \ell$ and $\alpha_{I E}$, guarantees that the segment II is in LD phase while III is in MC phase. From Eqn. (38), we get the maximum physically admissible value of the $\alpha_{1}$ to get both the segments I and II in LD phase and from condition (56),

$$
\beta>\frac{1}{\sqrt{\ell}+1}
$$

The expressions of canonical and non-canonical flux for $\mathrm{LD} / \mathrm{LD} / \mathrm{MC}$ phase are given by

$$
J_{1}=J_{s e g 1}=J_{s e g 2}=\frac{\alpha_{e f f 2}\left(W_{s}-\alpha_{e f f 2}\right)}{\left(W_{s}+\alpha_{e f f 2}(\ell-1)\right)},
$$

and

$$
J_{2}=\frac{1}{(\sqrt{\ell}+1)^{2}}-\frac{\alpha_{e f f 2}\left(W_{s}-\alpha_{e f f 2}\right)}{\left(W_{s}+\alpha_{e f f 2}(\ell-1)\right)},
$$

respectively, where $\alpha_{e f f 2}$ is given by 33 .

\section{Phase 4: $\mathrm{HD} / \mathrm{HD} / \mathrm{HD}$ phase}

In terms of the effective rates, the $\mathrm{HD} / \mathrm{HD} / \mathrm{HD}$ phase is specified by the conditions,

$$
\begin{aligned}
\beta_{\text {eff } 1}<\frac{1}{\sqrt{\ell}+1}, & \beta_{\text {eff } 1}<\alpha_{1}, \\
\beta_{\text {eff } 2}<\frac{W_{s}}{\sqrt{\ell}+1}, & \beta_{\text {eff } 2}<\alpha_{\text {eff } 2,}, \\
\beta & <\frac{1}{\sqrt{\ell}+1}, \quad \beta<\alpha_{\text {eff } 3 .}
\end{aligned}
$$

The corresponding fluxes of the rods in the three segments are

$$
\begin{aligned}
& J_{s e g 1}=\frac{\beta_{e f f 1}\left(1-\beta_{e f f 1}\right)}{1+\beta_{e f f 1}(\ell-1)}, \quad(a) \\
& J_{s e g 2}=\frac{\beta_{e f f 2}\left(W_{s}-\beta_{e f f 2}\right)}{W_{s}+\beta_{e f f 2}(\ell-1)}, \quad(b) \\
& J_{s e g 3}=\frac{\beta(1-\beta)}{1+\beta(\ell-1)}, \quad(c)
\end{aligned}
$$

while, the corresponding coverage densities are

$$
\begin{aligned}
& \rho_{c 1}=1-\beta_{\text {eff } 1}, \quad(a) \\
& \rho_{c 2}=1-\left(\beta_{\text {eff } 2} / W_{s}\right), \quad(b) \\
& \rho_{c 3}=1-\beta . \quad(c)
\end{aligned}
$$

Imposing the condition (15), i.e., equating the fluxes of segment II and III we get, 


$$
\frac{\beta(1-\beta)}{1+\beta(\ell-1)}=\frac{\beta_{e f f 2}\left(W_{s}-\beta_{e f f 2}\right)}{W_{s}+\beta_{e f f 2}(\ell-1)}+\alpha_{I E} P(\underbrace{0, \ldots, 0}_{\ell}),
$$

for $\mathrm{HD} / \mathrm{HD} / \mathrm{HD}$ phase (see Eq. A9 in the Appendix),

$$
P(\underbrace{0, \ldots, 0}_{\ell})=\frac{2 \beta}{2 \beta\left(1+\beta^{\prime}\right)+(1-\beta) \ell\left(2+\beta^{\prime}\right)} .
$$

where, $\beta^{\prime}=(1-\beta)(\ell-1)$.

Substituting Eq. (69), in Eq. (68) it becomes,

$$
\begin{array}{r}
\frac{\beta(1-\beta)}{1+\beta(\ell-1)}=\frac{\beta_{\text {eff } 2}\left(W_{s}-\beta_{\text {eff } 2}\right)}{W_{s}+\beta_{\text {eff } 2}(\ell-1)} \\
+\frac{2 \alpha_{I E} \beta}{2 \beta\left(1+\beta^{\prime}\right)+(1-\beta) \ell\left(2+\beta^{\prime}\right)}
\end{array} .
$$

Solving this quadratic equation 70 we get the expression of $\beta_{\text {eff } 2}$, in terms of $\beta, W_{s}$ and $\ell$ :

$$
\beta_{e f f 2}=\frac{-h_{2}-\sqrt{h_{2}^{2}-4 h_{1} h_{3}}}{2 h_{1}}
$$

the general expressions for $h_{1}, h_{2}$ and $h_{3}$ are given in the appendix D (see Eq. D2, D3 and D4). Now imposing the condition (14), i.e. , equating (49)(a) and 49)(b), we get

$$
\frac{\beta_{e f f 1}\left(1-\beta_{e f f 1}\right)}{1+\beta_{e f f 1}(\ell-1)}=\frac{\beta_{e f f 2}\left(W_{s}-\beta_{e f f 2}\right)}{W_{s}+\beta_{e f f 2}(\ell-1)},
$$

solving which for the unknown $\beta_{\text {eff } 1}$ we get,

$$
\beta_{\text {eff } 1}=\frac{-q_{1}-\sqrt{q_{1}^{2}-4 q_{2} q_{3}}}{2 q_{3}}
$$

the general expressions of $q_{1}, q_{2}$ and $q_{3}$ are given in appendix D (see Eq. D6, D7 and D8). Substituting Eqn. (71) into (72), we get the expression for $\beta_{\text {eff } 1}$, in terms of $\beta, W_{s}$ and $\ell$. Thus, the expressions for canonical and non-canonical flux for $\mathrm{HD} / \mathrm{HD} / \mathrm{HD}$ phase are given by

$$
\begin{aligned}
& J_{1}=J_{\text {seg } 1}=J_{\text {seg } 2}=\frac{\beta_{\text {eff } 2}\left(W_{s}-\beta_{e f f 2}\right)}{W_{s}+\beta_{\text {eff } 2}(\ell-1)}, \\
& \text { and } \\
& J_{2}=\frac{\beta(1-\beta)}{1+\beta(\ell-1)}-\frac{\beta_{e f f 2}\left(W_{s}-\beta_{e f f 2}\right)}{W_{s}+\beta_{\text {eff } 2}(\ell-1)} .
\end{aligned}
$$

respectively.

Phase 5: HD/MC/MC phase This phase can be specified by the following conditions,

$$
\begin{array}{rlrl}
\beta_{e f f 1} & <\frac{1}{\sqrt{\ell}+1}, & & \beta_{e f f 1}<\alpha_{1}, \\
\beta_{e f f 2}>\frac{W_{s}}{\sqrt{\ell}+1}, & \alpha_{e f f 2}>\frac{W_{s}}{\sqrt{\ell}+1}, \\
\beta & >\frac{1}{\sqrt{\ell}+1}, & \alpha_{e f f 3} & >\frac{1}{\sqrt{\ell}+1} .
\end{array}
$$

The fluxes of the rods that follow from equations 10 (a) and 8 (a), are given by

$$
\begin{aligned}
& J_{\text {seg } 1}=\frac{\beta_{\text {eff } 1}\left(1-\beta_{\text {eff } 1}\right)}{1+\beta_{\text {eff } 1}(\ell-1)}, \quad(a) \\
& J_{\text {seg } 2}=\frac{W_{s}}{(\sqrt{\ell}+1)^{2}}, \quad(b) \\
& J_{\text {seg } 3}=\frac{1}{(\sqrt{\ell}+1)^{2}}, \quad(c)
\end{aligned}
$$

while, from the equations (10)(b) and 8)(b), the corresponding coverage densities are given by

$$
\begin{aligned}
& \rho_{c 1}=1-\beta_{\text {eff } 1}, \quad(a) \\
& \rho_{c 2}=\frac{\sqrt{\ell}}{\sqrt{\ell}+1}, \quad(b) \\
& \rho_{c 3}=\frac{\sqrt{\ell}}{\sqrt{\ell}+1} . \quad(c)
\end{aligned}
$$

Now imposing the condition (14), i.e., equating (76) (a) and 76 (b), we get

$$
\frac{\beta_{e f f 1}\left(1-\beta_{e f f 1}\right)}{1+\beta_{e f f 1}(\ell-1)}=\frac{W_{s}}{(\sqrt{\ell}+1)^{2}},
$$

solving which for $\beta_{\text {eff } 1}$ we get

$$
\beta_{e f f 1}=\frac{-k_{1}-\sqrt{\left(k_{1}\right)^{2}+4 k_{2}}}{2 k_{3}},
$$

where,

$$
\begin{aligned}
& k_{1}=1+W_{s}-W_{s} \ell+\ell+2 \sqrt{\ell}, \\
& k_{2}=(-\ell-2 \sqrt{\ell}-1) W_{s}, \\
& k_{3}=(-\ell-2 \sqrt{\ell}-1) .
\end{aligned}
$$

From condition 75 it follows that the $\mathrm{HD} / \mathrm{MC} / \mathrm{MC}$ phase exists on the $\alpha_{1}-\beta$ plane in the region where

$$
\begin{aligned}
\alpha_{1} & >\frac{-k_{1}-\sqrt{\left(k_{1}\right)^{2}+4 k_{2}}}{2 k_{3}}, \\
\beta & >\frac{1}{\sqrt{\ell}+1} .
\end{aligned}
$$


The corresponding canonical and non-canonical flux in the $\mathrm{HD} / \mathrm{MC} / \mathrm{MC}$ phase are

$$
J_{1}=J_{\text {seg } 1}=J_{\text {seg } 2}=\frac{W_{s}}{(\sqrt{\ell}+1)^{2}},
$$

and

$$
J_{2}=\frac{1}{(\sqrt{\ell}+1)^{2}}-\frac{W_{s}}{(\sqrt{\ell}+1)^{2}} .
$$

respectively.

\section{Phase 6: HD/MC/LD phase}

This phase can be specified by the following conditions:

$$
\begin{array}{ll}
\beta_{e f f 1}<\frac{1}{\sqrt{\ell}+1}, & \beta_{\text {eff } 1}<\alpha_{1}, \\
\beta_{e f f 2}>\frac{W_{s}}{\sqrt{\ell}+1}, & \alpha_{e f f 2}>\frac{W_{s}}{\sqrt{\ell}+1}, \\
\alpha_{e f f 3}<\frac{1}{\sqrt{\ell}+1}, & \alpha_{e f f 3}<\beta .
\end{array}
$$

Under steady state condition the fluxes of the rods that follow from equations $(10 \mathrm{a}),(8 \mathrm{a})$ and $(9 \mathrm{a})$, are given by

$$
\begin{aligned}
& J_{\text {seg } 1}=\frac{\beta_{\text {eff } 1}\left(1-\beta_{\text {eff } 1}\right)}{1+\beta_{\text {eff } 1}(\ell-1)}, \\
& J_{\text {seg } 2}=\frac{W_{s}}{(\sqrt{\ell}+1)^{2}}, \quad(b) \\
& J_{\text {seg } 3}=\frac{\alpha_{e f f 3}\left(1-\alpha_{e f f 3}\right)}{1+\alpha_{\text {eff } 3}(\ell-1)},
\end{aligned}
$$

while, from the equations $(10 \mathrm{~b}),(8 \mathrm{~b})$ and $(9 \mathrm{~b})$, the corresponding coverage densities are given by,

$$
\begin{aligned}
\rho_{c 1} & =1-\beta_{\text {eff } 1}, \quad(a) \\
\rho_{c 2} & =\frac{\sqrt{\ell}}{\sqrt{\ell}+1}, \quad(b) \\
\rho_{c 3} & =\frac{\alpha_{e f f 3} \ell}{1+\alpha_{e f f 3}(\ell-1)} . \quad(c)
\end{aligned}
$$

Now imposing the condition (14), i.e., equating (84)(a) and 84 (b), we get a quadratic equation for $\beta_{\text {eff } 1 \text { whose }}$ solution is given by (79). Next, using Eq. (15), i.e., equating the fluxes of segment II and III we get

$$
\frac{\alpha_{e f f 3}\left(1-\alpha_{e f f 3}\right)}{1+\alpha_{e f f 3}(\ell-1)}=\frac{W_{s}}{(\sqrt{\ell}+1)^{2}}+\alpha_{I E} P(\underbrace{0, \ldots, 0}_{\ell}),
$$

for $\mathrm{HD} / \mathrm{MC} / \mathrm{LD}$ phase (see Eq. A7 in the Appendix)

$$
P(\underbrace{0, \ldots, 0}_{\ell})=\frac{2\left(1-\alpha_{e f f 3}\right)}{2\left(1-\alpha_{e f f 3}\right)\left(1+\alpha_{e f f 3}^{\prime}\right)+\alpha_{e f f 3} \ell\left(2+\alpha_{e f f 3}^{\prime}\right)},
$$

where, $\alpha_{\text {eff } 3}^{\prime}=\alpha_{\text {eff } 3}(\ell-1)$.

Since, we are taking the special site $i_{s}$, far from the boundary between segment II and III, $\alpha_{N I E}$ is not affected by $\alpha_{I E}$. Therefore, to simplify the calculation, we first solve equation (86) for $\alpha_{I E}=0$ getting the expression for $\alpha_{e f f 3}=\alpha_{N I E}$, in terms of $W_{s}$ and $\ell$, to be

$$
\alpha_{N I E}=\frac{-k_{1}-\sqrt{\left(k_{1}\right)^{2}+4 k_{2}}}{2 k_{3}},
$$

and hence in the general case of of $\alpha_{I E} \neq 0, \alpha_{e f f 3}=$ $\alpha_{N I E}+\alpha_{I E}$, i.e.,

$$
\alpha_{e f f 3}=\frac{-k_{1}-\sqrt{\left(k_{1}\right)^{2}+4 k_{2}}}{2 k_{3}}+\alpha_{I E} .
$$

From condition 83 , we find that the segment III would be in LD phase for

$$
\alpha_{N I E}+\alpha_{I E}<\frac{1}{(\sqrt{\ell}+1)} .
$$

But, since, the Eqn. 90 is independent of $\alpha_{1}$ this phase cannot be displayed on the $\alpha_{1}-\beta$ plane in case of signalindependent initiation. However, in the case of signaldependent initiation

$$
\alpha_{I E}=\alpha-\alpha_{1}
$$

the HD/MC/LD phase appears on the $\alpha_{1}-\beta$ plane in the region where $\left(\alpha_{N I E}+\alpha-\alpha_{1}\right)<1 /(\sqrt{\ell}+1)$, i.e.,

$$
\begin{aligned}
& \alpha_{1}>\alpha+\frac{-k_{1}-\sqrt{\left(k_{1}\right)^{2}+4 k_{2}}}{2 k_{3}}-\frac{1}{(\sqrt{\ell}+1)}, \\
& \beta>\frac{-k_{1}-\sqrt{\left(k_{1}\right)^{2}+4 k_{2}}}{2 k_{3}}+\alpha-\alpha_{1} .
\end{aligned}
$$

For a given $\alpha_{1}$, when $\alpha_{1}>\alpha+\alpha_{N I E}-1 /(\sqrt{\ell}+1)$, if $\beta$ is increased so that it exceeds $\alpha_{\text {eff } 3}$ the segment III transforms to LD phase from HD phase and the composite phase of the system makes a transition from $\mathrm{HD} / \mathrm{MC} / \mathrm{HD}$ to $\mathrm{HD} / \mathrm{MC} / \mathrm{LD}$. Similarly, For a given $\beta$, when $\beta>1 /(\sqrt{\ell}+1)$, decrease of $\alpha_{1}$ or increase of $\alpha_{I E}$ can result in a value of $\alpha_{e f f 3}$ that just exceeds $1 /(\sqrt{\ell}+1)$. At this point a transition of segment III from LD phase to MC phase takes place and, consequently, the system, as a whole, exhibits a transition from composite phase $\mathrm{HD} / \mathrm{MC} / \mathrm{LD}$ to $\mathrm{HD} / \mathrm{MC} / \mathrm{MC}$.

Finally, the canonical and non-canonical fluxes in the $\mathrm{HD} / \mathrm{MC} / \mathrm{LD}$ phase are

$$
\begin{aligned}
& J_{1}=J_{\text {seg } 1}=J_{\text {seg } 2}=\frac{W_{s}}{(\sqrt{\ell}+1)^{2}}, \\
& J_{2}=\frac{\alpha_{e f f 3}\left(1-\alpha_{e f f 3}\right)}{1+\alpha_{e f f 3}(\ell-1)}-\frac{W_{s}}{(\sqrt{\ell}+1)^{2}} .
\end{aligned}
$$

Phase 7: HD/MC/HD phase This phase can be specified by the following conditions, 
(a)

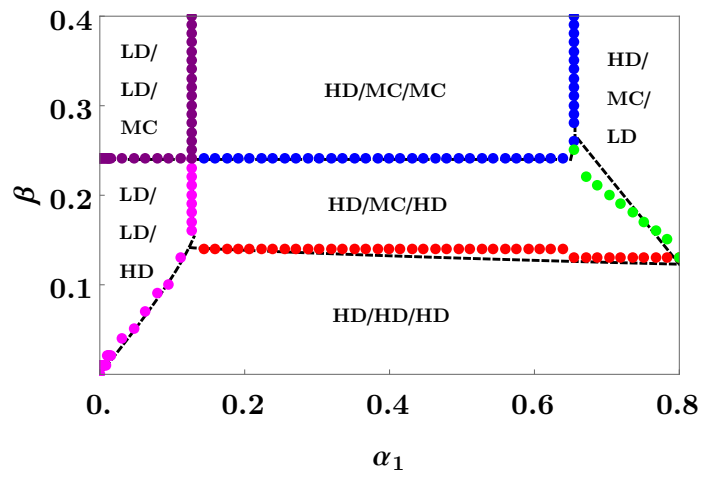

(b)

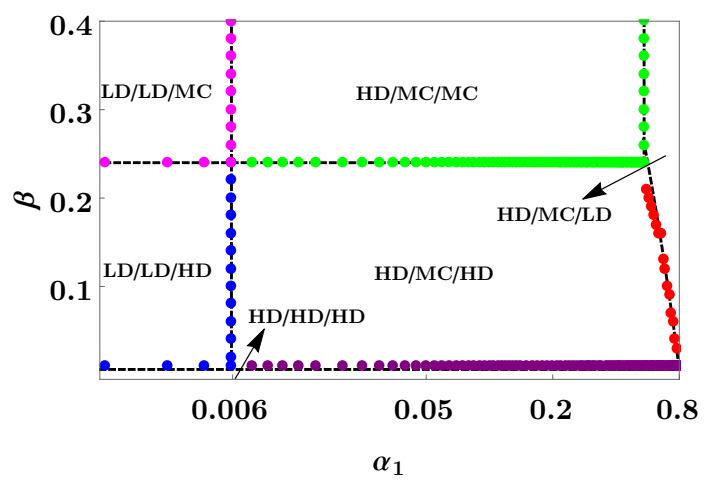

FIG. 3. (Color online) Phase diagrams of the model in the $\alpha_{1}-\beta$ plane, in the case of signal-dependent initiation, are plotted for (a) $\gamma=0.9$, and (b) $\gamma=0.1$. The theoretical prediction obtained under MFA are drawn by dashed curves while the numerical data obtained from Monte-Carlo simulations are shown by discrete symbols. The numerical values of the relevant parameters used here are $L+\ell-1=1200+\ell-1$, $\ell=10, i_{s}=530, n=3, m=5, \alpha=0.8$ and $W=1$.

$$
\begin{array}{rlrl}
\beta_{e f f 1} & <\frac{1}{\sqrt{\ell}+1}, & \beta_{\text {eff } 1}<\alpha_{1}, \\
\beta_{\text {eff } 2}>\frac{W_{s}}{\sqrt{\ell}+1}, & \alpha_{\text {eff } 2}>\frac{W_{s}}{\sqrt{\ell}+1}, \\
\beta<\frac{1}{\sqrt{\ell}+1}, & \beta<\alpha_{\text {eff } 3 .}
\end{array}
$$

Under stationary state condition the fluxes of the rods that follow from equations (10 a) and 8) a), are given by

$$
\begin{aligned}
J_{\text {seg } 1} & =\frac{\beta_{e f f 1}\left(1-\beta_{e f f 1}\right)}{1+\beta_{\text {eff } 1}(\ell-1)}, \\
J_{\text {seg } 2} & =\frac{W_{s}}{(\sqrt{\ell}+1)^{2}}, \quad(b) \\
J_{\text {seg } 3} & =\frac{\beta(1-\beta)}{1+\beta(\ell-1)}, \quad(c)
\end{aligned}
$$

while, from the equations $(10 \mathrm{~b})$ and $(8 \mathrm{~b})$, the corresponding coverage densities are given by,
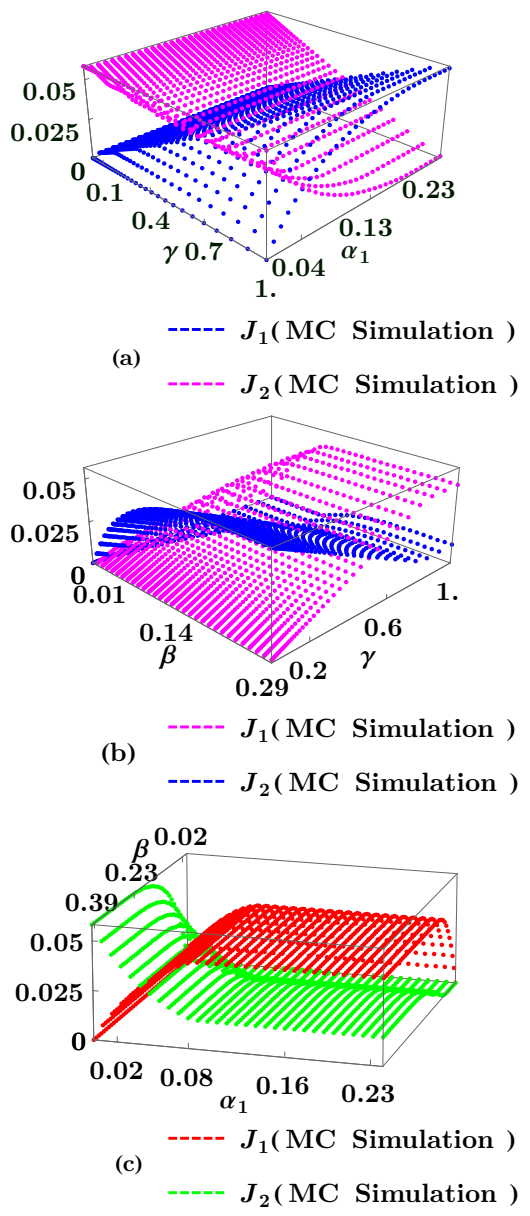

FIG. 4. (Color online) 3D plots of the fluxes $J_{1}$ and $J_{2}$ of the $\operatorname{rod}_{1}$ and $\operatorname{rod}_{2}$, respectively, against (a) $\alpha_{1}$ and $\gamma$ (for constant $\beta=0.8$ ), (b) $\gamma$ and $\beta$ (for constant $s=0.1$ ), (c) $\alpha_{1}$ and $\beta$ (for constant $\gamma=0.47$ ), respectively, in the case of signal-dependent initiation. The numerical values of the other relevant parameters used in this figure are $L+\ell-1=$ $1200+\ell-1, \ell=10, i_{s}=512, n=1, m=7, W=1$ and $\alpha=0.8$.

$$
\begin{aligned}
\rho_{c 1} & =1-\beta_{\text {eff } 1}, \quad(a) \\
\rho_{c 2} & =\frac{\sqrt{\ell}}{\sqrt{\ell}+1}, \quad(b) \\
\rho_{c 3} & =1-\beta . \quad(c)
\end{aligned}
$$

Now imposing the condition (14), i.e., equating (95) (a) and 95 (b), we get a quadratic equation for $\beta_{\text {eff } 1}$ whose solution is given by $(79)$. Imposing Eq. (15) we now get

$$
\frac{\beta(1-\beta)}{1+\beta(\ell-1)}=\frac{W_{s}}{(\sqrt{\ell}+1)^{2}}+\alpha_{I E} P(\underbrace{0, \ldots, 0}_{\ell}),
$$

where substitution of

$$
P(\underbrace{0, \ldots, 0}_{\ell})=\frac{2 \beta}{2 \beta\left(1+\beta^{\prime}\right)+(1-\beta) \ell\left(2+\beta^{\prime}\right)},
$$




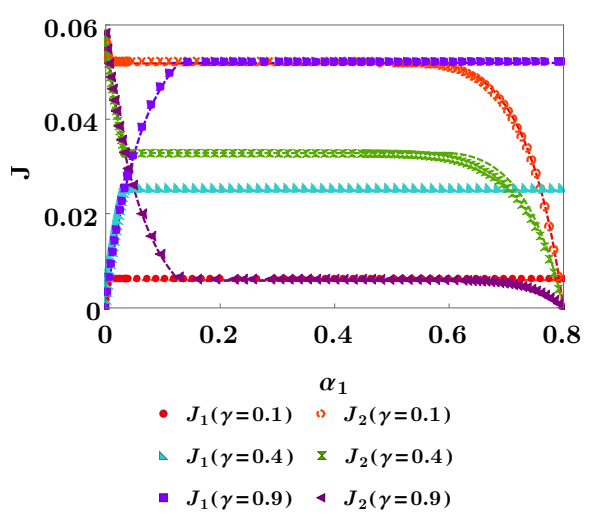

FIG. 5. (Color online) Two-dimensional cross sections of Fig. (4(a)) corresponding to three different values of $\gamma$ are plotted. Thus, fluxes $J_{1}$ and $J_{2}$ are plotted against $\alpha_{1}$ for three different values of $\gamma$. The theoretical predictions obtained under MFA are drawn with continuous curves and the numerical data obtained from Monte-Carlo simulations are shown with discrete symbols. The numerical values of the other relevant parameters used in the figure are $L+\ell-1=1200+\ell-1$, $\ell=10, i_{s}=512, n=1, m=7, W=1, \alpha=0.8$ and $\beta=0.8$.

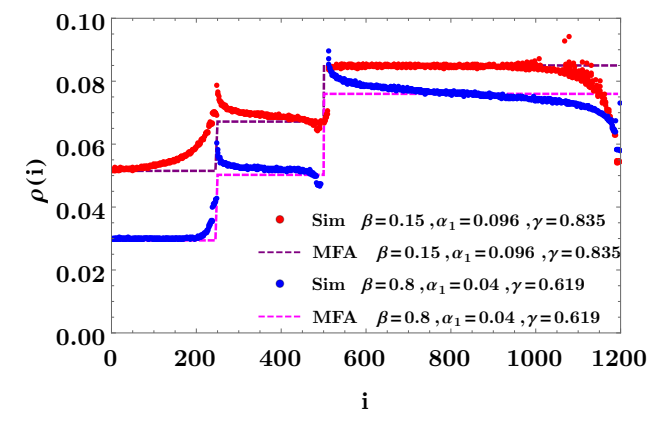

FIG. 6. (Color online) Density profiles of rods $(\rho(i)=$ $\left.P_{1}(i)+P_{2}(i)\right)$ in the case of signal-dependent initiation are plotted for two sets of values of $\beta, \alpha_{1}$ and $\gamma$. The theoretical prediction obtained under MFA are drawn by continues curves and numerical data obtained from Monte-Carlo simulations are shown with discrete symbols. The numerical values of the other relevant parameters used in this figure are $L+\ell-1=1200+\ell-1, \ell=10, i_{s}=512, n=1, m=7$, $W=1$ and $\alpha=0.8$. The blue curve corresponds to the $\mathrm{LD} / \mathrm{LD} / \mathrm{MC}$ phase where as the red curve corresponds to the $\mathrm{LD} / \mathrm{LD} / \mathrm{HD}$ phase.

for $\mathrm{HD} / \mathrm{MC} / \mathrm{HD}$ phase (see the derivation of Eq. A9 in the Appendix), with $\beta^{\prime}=(1-\beta)(\ell-1)$, we get an equation whose solution yields the expression for $\beta$ in terms of $W_{s}, \alpha_{I E}$ and $\ell$.

Solving this quartic equation of $\beta$, we get the expression of $\beta$ in terms of $W_{s}, \ell$ and $\alpha_{I E}$. Out of the four solutions of the quartic equation, two are the negative and, therefore, not physically admissible. Out of two positive (a)

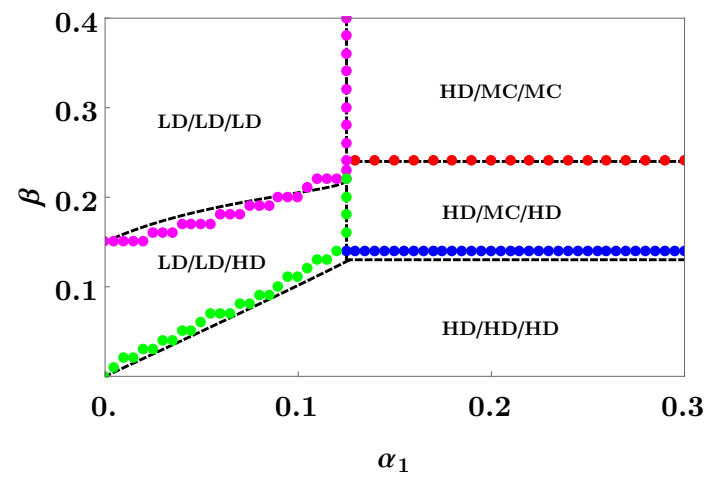

(b)

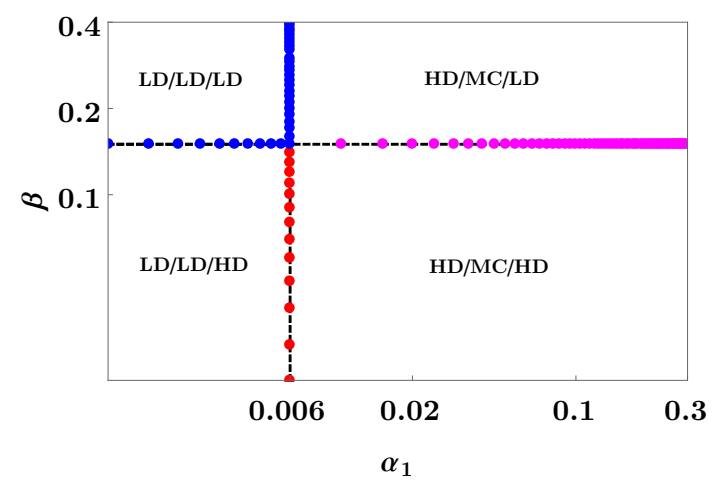

FIG. 7. (Color online) Phase diagrams of the model in the case of signal-independent initiation are plotted keeping $\gamma=0.9$ and $\alpha_{I E}=0.15$, fixed in (a) and $\gamma=0.1$ and $\alpha_{I E}=0.15$ fixed in (b). The theoretical prediction obtained under MFA are drawn by continues curves and numerical data obtained from Monte-Carlo simulations are shown with discrete symbols. The numerical values of the other relevant parameters used in this figure are $L+\ell-1=1200+\ell-1$, $\ell=10, i_{s}=512, n=1, m=7$, and $W=1$.

solutions we have chosen the one with the negative sign to be the physical solution because, as restricted by (94), $\beta$ must be less than $1 /(\sqrt{\ell}+1)$. The general expression is too complicated to be reproduced here. Instead, the solution for a specific set of parameter values, namely, $W_{s}=0.9$ and $\ell=10$, is given in the appendix $\mathrm{E}$.

From condition (94) occurrence of the HD/MC/HD phase requires

$$
\begin{aligned}
\alpha_{1} & >\frac{-k_{1}-\sqrt{\left(k_{1}\right)^{2}+4 k_{2}}}{2 k_{3}} \\
\frac{1}{(\sqrt{\ell}+1)} & >\beta>\left(z_{0}-0.5 \sqrt{z_{1}+\frac{z_{2}}{z_{3}}+z_{4}}\right. \\
& \left.-0.5 \sqrt{z_{5}-\frac{z_{6}}{z_{7}}-z_{8}-\frac{z_{9}}{z_{10}}}\right)
\end{aligned}
$$

(As example, for the expressions of $z_{\mu}(\mu=0,1, \cdots 10)$ see appendix E ) For any given $\alpha_{1}$ that satisfies the condition $\alpha_{1}>\left(-k_{1}-\sqrt{\left(k_{1}\right)^{2}+4 k_{2}}\right) /\left(2 k_{3}\right)$, the phase 
(a)

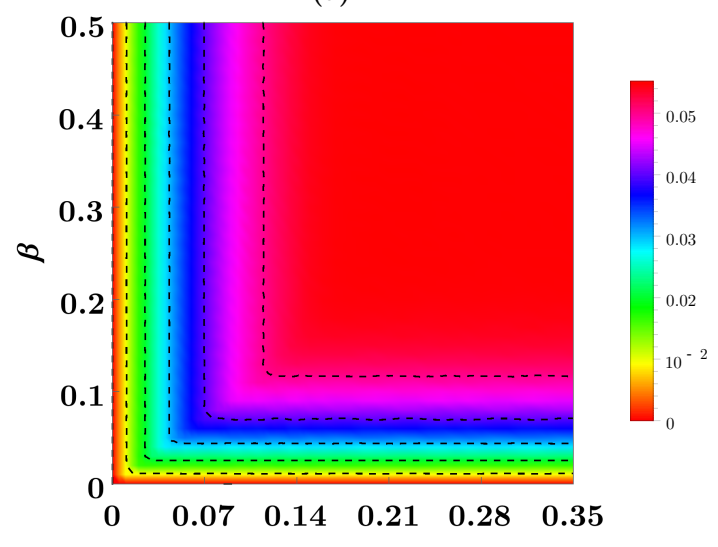

$\alpha_{1}$

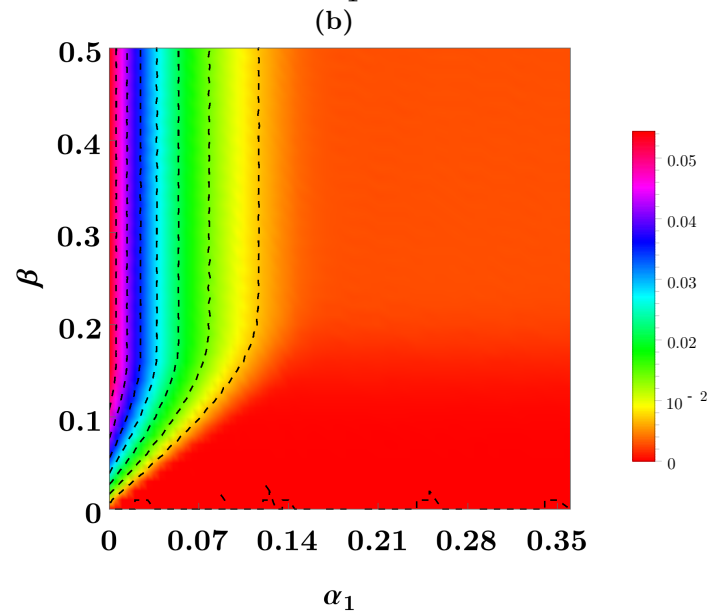

FIG. 8. (Color online) Contour plots of the fluxes $J_{1}$ and $J_{2}$ in the $\alpha_{1}-\beta$ plane shown in (a) and (b) are obtained from Monte Carlo simulations of the model in the case of signalindependent initiation. The numerical values of the other relevant parameters are $L+\ell-1=1200+\ell-1, \ell=10$, $i_{s}=512, n=1, m=7, W=1, \alpha_{I E}=0.35$ and $\gamma=0.74$.

in the segment III transforms from HD phase to MC phase when $\beta$ just exceeds the value $1 /(\sqrt{\ell}+1)$ and the composite phase of the system makes a transition from $\mathrm{HD} / \mathrm{MC} / \mathrm{HD}$ to $\mathrm{HD} / \mathrm{MC} / \mathrm{MC}$. On the other hand, as $\beta$ decreases, the phase in segment II transforms from MC phase to HD phase at $\beta=\left(z_{0}-0.5 \sqrt{z_{1}+\left(z_{2} / z_{3}\right)+z_{4}}-\right.$ $\left.0.5 \sqrt{z_{5}-\left(z_{6} / z_{7}\right)-z_{8}-\left(z_{9} / z_{10}\right)}\right)$ and the composite phase of the system makes a transition from $\mathrm{HD} / \mathrm{MC} / \mathrm{HD}$ to $\mathrm{HD} / \mathrm{HD} / \mathrm{HD}$. The physical reason for this transition is that, below this critical value of $\beta$, the segment III creates an effective bottleneck at the end of the segment II for the rods moving forward in segment II.

The canonical and non-canonical fluxes in the $\mathrm{HD} / \mathrm{MC} / \mathrm{HD}$ phase are (a)

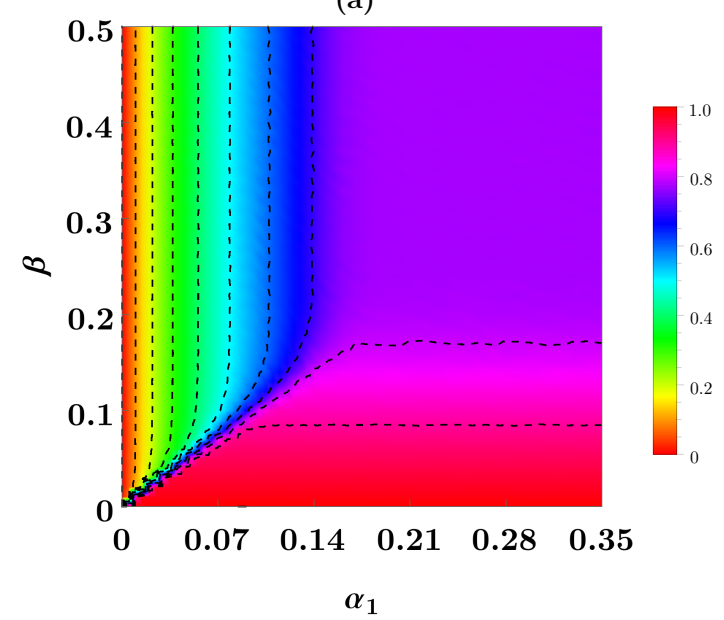

(b)

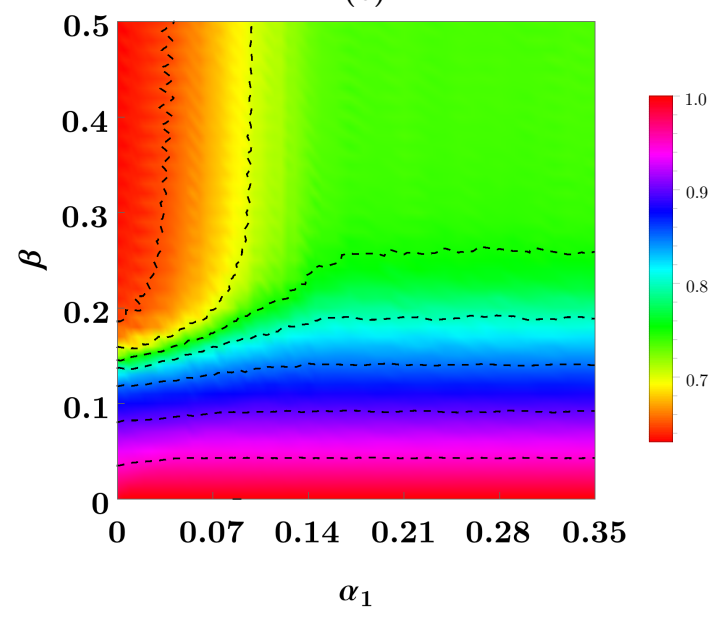

FIG. 9. (Color online) Contour plots of the coverage densities in the segments I and III in the $\alpha_{1}-\beta$ plane shown in (a) and (b) are obtained from Monte Carlo simulations of the model in the case of signal-independent initiation. The numerical values of the other relevant parameters are $L+\ell-1=1200+$ $\ell-1, \ell=10, i_{s}=512, n=1, m=7, W=1, \alpha_{I E}=0.35$ and $\gamma=0.74$.

$$
\begin{aligned}
& J_{1}=J_{\text {seg } 1}=J_{\text {seg } 2}=\frac{W_{s}}{(\sqrt{\ell}+1)^{2}}, \\
& J_{2}=\frac{\beta(1-\beta)}{1+\beta(\ell-1)}-\frac{W_{s}}{(\sqrt{\ell}+1)^{2}} .
\end{aligned}
$$

\section{B. Graphical display of results}

In two separate subsections below we present the results in the two scenarios, namely signal-dependent and signal-indepedent initiations. 


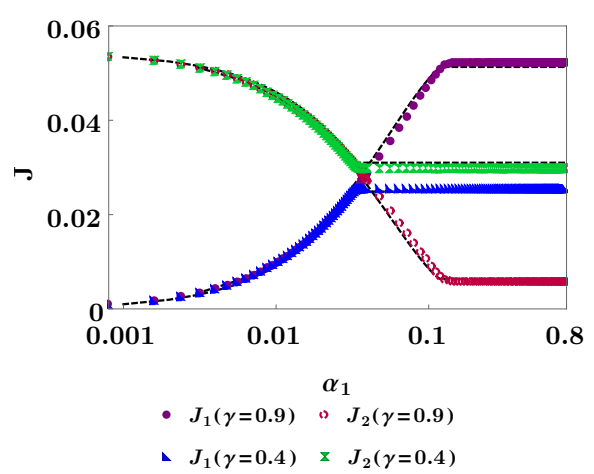

FIG. 10. (Color online) Fluxes $J_{1}$ and $J_{2}$ in the case of signalindependent initiation are plotted against $\alpha_{1}$, for two different values of $\gamma$ and for a fixed $\beta$. The theoretical prediction obtained under MFA are drawn by continues curves and numerical data obtained from Monte-Carlo simulations are shown with discrete symbols. The numerical values of the other relevant parameters used in this figure are $L+\ell-1=1200+\ell-1$, $\ell=10, i_{s}=512, n=1, m=7, W=1$ and $\beta=0.8$.

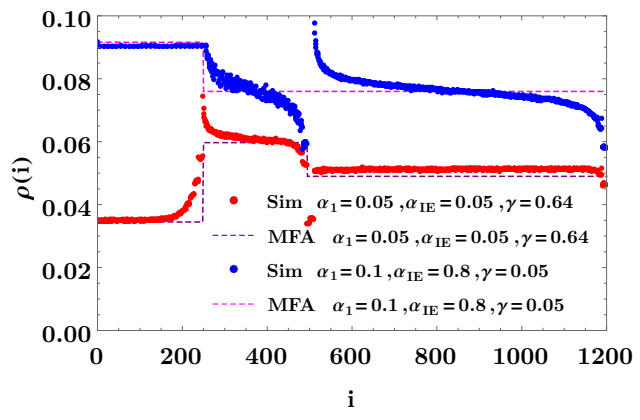

FIG. 11. (Color online) Density profiles of rods $(\rho(i)=$ $\left.P_{1}(i)+P_{2}(i)\right)$ are plotted for two sets of values of the parameters $\alpha_{1}, \alpha_{I E}$ and $\gamma$. The theoretical prediction obtained under MFA are drawn by continues curves and numerical data obtained from Monte-Carlo simulations are shown with discrete symbols. The numerical values of the other relevant parameters used in this figure are $L+\ell-1=1200+\ell-1$, $\ell=10, i_{s}=512, n=1, m=7, \beta=0.8$ and $W=1$.

\section{Signal Dependent Initiation}

In Fig. (3) (a) and (b) we present the phase diagrams of the system in $\alpha_{1}-\beta$ plane for $\gamma=0.9$ and $\gamma=0.1$, respectively. The reason for taking two extreme limits of $\gamma$ for the two phase diagrams is to highlight how the phase boundaries get drastically affected by the variation of the hopping $W_{s}$ in the extended bottleneck region. Our theoretical predictions, based on MFA, are in good agreement with the corresponding numerical data obtained from $\mathrm{MC}$ simulations for all phase boundaries except that between the $\mathrm{HD} / \mathrm{MC} / \mathrm{LD}$ and $\mathrm{HD} / \mathrm{MC} / \mathrm{HD}$ phases.

The line separating the $\mathrm{LD} / \mathrm{LD} / \mathrm{HD}$ phase from
$\mathrm{HD} / \mathrm{HD} / \mathrm{HD}$ phase is given by the expression (51). The transition from $\mathrm{LD} / \mathrm{LD} / \mathrm{HD}$ phase to the $\mathrm{HD} / \mathrm{HD} / \mathrm{HD}$ phase is accompanied by a discontinues change in the coverage density of segment I and II while that of segment III remains unaltered. Similarly, expression 92 represents the line that separates the $\mathrm{HD} / \mathrm{MC} / \mathrm{HD}$ phase from $\mathrm{HD} / \mathrm{MC} / \mathrm{LD}$ phase; during the transition across this line the coverage density of only the segment III changes discontinuously.

In Fig. (4) we present 3D plots of the fluxes $J_{1}$ and $J_{2}$ as a function of (a) $\alpha_{1}$ and $\gamma$ at a fixed value of $\beta$, (b) $\gamma$ and $\beta$ at a fixed value of $s$, (c) $\alpha_{1}$ and $\beta$ for a fixed value of $\gamma$. One common feature of all the three 3D plots is that while one flux (say $J_{1}$ ) increases then the other, namely $J_{2}$, decreases and the two surfaces cross each other. The suppression of $J_{2}$ by high value of the flux $J_{1}$ is displayed directly in the $2 \mathrm{D}$ cross sections of Fig. (4) (a) for different fixed values of $\gamma$ as shown in Fig.5. As $\gamma$ decreases, the areas on the $\alpha_{1}-\beta$ plane covered by all the three phases, namely, LD/LD/MC, $\mathrm{LD} / \mathrm{LD} / \mathrm{HD}$ and $\mathrm{HD} / \mathrm{HD} / \mathrm{HD}$, shrink.

The density profiles of the rods $\left(\rho(i)=P_{1}(i)+P_{2}(i)\right)$ are plotted in Fig. (6) for two sets of values of the parameters $\beta, \alpha_{1}, \gamma$. The blue and red curves corresponds to the $\mathrm{LD} / \mathrm{LD} / \mathrm{MC}$ phase and $\mathrm{LD} / \mathrm{LD} / \mathrm{HD}$ phase, respectively. As is well known, the MFA is not as accurate for $\mathrm{MC}$ phase as it is for the LD and HD phases.

\section{Signal Independent Initiation}

The suppression of $J_{2}$ by $J_{1}$ is observed also in the case of signal-independent initiation just as the same phenomenon was observed in the case of signal-dependent initiation.Thus, this switch-like regulation of the fluxes of two mutually interfering species of rods seems to be an ubiquitous feature of the co-directional two-species exclusion process with two distinct sites of entry for the respective species (see Fig.7 for phase diagram).

The contour plots of fluxes and coverage densities in Figs 8 and 9 respectively, are consistent with the other results described above (see also Figs 10 and 11 .

\section{SUMMARY AND CONCLUSION}

We have developed a two-species exclusion process where each of the two species of hard rods have their distinct pair of entry and exit sites. An extended bottleneck exists in between the entry sites for the two species of rods; one entry site is located immediately downstream from the bottleneck while the other entry site is located far upstream from it. The static bottleneck is characterized by relatively slower rate of forward hopping of the rods in this region compared to that in the other regions. The number of species of the rods and the relative locations of their entry sites with respect to that of the 
bottleneck are motivated by an unconventional mode of gene translation via IRES.

In principle, the results on the collective spatiotemporal organization of the rods in this exclusion model may have implications for regulation of unconventional translation. However, the work reported here is not intended to account for experimental data for any specific system. Instead, the main emphasis has been to explore the generic features of the composite phases, fluxes and density profiles that are displayed by this simple model. More specifically we demonstrate how the boundaries between the various phases shift with the variation of some of the key model parameters that, at least in principle, can be varied in a controlled manner in experiments. But, out of all the seven possible phases, only a few may be realizable under physiological conditions unless a living cell has internal mechanism for altering the structure and/or stability of the secondary structure of the mRNA under different circumstances.

We have carried out our theoretical analysis in two distinct scenarios that correspond, respectively, to signaldependent- and signal-independent IRES. Interestingly, in both scenarios we have observed switch-like regulation of the fluxes of the two species of rods: a sufficiently high-level of flux of one species can suppress that of the other to a low level. This phenomenon, that we propose to name as "translational interference", may be regarded as an analog of the well known phenomenon of "transcriptional interference" observed in the transcription of genetic message, encoded on DNA, by another type of motor called RNA polymerase. However, there is a crucial difference between the two phenomena. Since mRNA track can form several types of secondary structures 104 these can create wide varieties of bottlenecks against translation whereas, to our knowledge, analogous bottlenecks are not created by any secondary structure of the DNA template in the transcriptional process. Therefore, an additional control is available in 'translational interference' as compared to 'transcriptional interference'.

\section{ACKNOWLEDGEMENT}

We thank Kavita Jain and Tanweer Hussain for useful comments and suggestions. This work has been supported by J.C. Bose National Fellowship (DC), "Prof. S. Sampath Chair" Professorship (DC) and by UGC Senior Research Fellowship (BM).
[1] M.R. Evans and T. Hanney, J. Phys. A: Math. Gen. 38, R195 (2005).

[2] R. Blythe and M.R. Evans, J. Phys. A: Math. Gen. 40, R333 (2007).

[3] G.M. Schütz, in: Phase Transitions and Critical Phenomena, eds. C. Domb and J.L. Lebowitz (Academic Press, 2001).

[4] B. Derrida, Phys. Rep. 301, 65-83 (1998).

[5] K. Mallick, Physica A 418, 17-48 (2015).

[6] D. Mukamel, in: Soft and Fragile matter: Nonequilibrium Dynamics, Metastability and Flow, eds. M.E. Cates and M.R. Evans, p.237 (CRC Press, 2000).

[7] D. Chowdhury, L. Santen and A. Schadschneider, Phys. Rep. 329, 199-329 (2000).

[8] A. Schadschneider, D. Chowdhury and K. Nishinari, Stochastic transport in complex systems: from molecules to vehicles, (Elsevier, 2010).

[9] D. Chowdhury, A. Schadschneiner and K. Nishinari, Phys. of Life Rev. 2, 318 (2005).

[10] T. Chou, K. Mallick and R.K.P. Zia, Rep. Prog. Phys. 74, 116601 (2011).

[11] D. Chowdhury, Phys. Rep. 529, 1 (2013).

[12] C. Appert-Rolland, M. Ebbinghaus and L. Santen, Phys. Rep. 593, 1-59 (2015).

[13] C. MacDonald, J. Gibbs and A. Pipkin, Biopolymers 6, 1 (1968).

[14] C. MacDonald and J. Gibbs, Biopolymers, 7, 707 (1969).

[15] T. Tripathi and D. Chowdhury, Phys. Rev. E 77, 011921 (2008).

[16] S. Klumpp and T. Hwa, Proc. Natl. Acad. Sci. 105, 18159, (2008).
[17] S. Klumpp, J. Stat. Phys. 142, 1252 (2011).

[18] M. Sahoo and S. Klumpp, EPL 96, 60004 (2011).

[19] Y. Ohta, T. Kodama and S. Ihara, Phys. Rev. E 84, 041922 (2011).

[20] J. Wang, B. Pfeuty, Q. Thommen, M.C. Romano and M. Lefranc, Phys. Rev. E 90, 050701(R) (2014).

[21] G.M. Schütz, J. Phys. A 36, R339 (2003).

[22] A. Kunwar, D. Chowdhury, A. Schadschneider and K. Nishinari, J. Stat. Mech: Theor. Expt. P06012 (2006).

[23] A. John, A. Schadschneider, D. Chowdhury and K. Nishinari, J. Theor. Biol. 231, 279 (2004).

[24] C. Lin, G. Steinberg and P. Ashwin, J. Stat. Mech. Theor. Expt. P09027 (2011).

[25] G. Lakatos and T. Chou, J. Phys. A: Math. Gen. 36, 2027 (2003).

[26] L. B. Shaw, R.K.P. Zia and K. H Lee, Phys. Rev. E 68, $021910(2003)$.

[27] L.B. Shaw, J.P. Sethna and K.H. Lee, Phys. Rev. E 70, 021901 (2004).

[28] L.B. Shaw, A.B. Kolomeisky and K.H. Lee, J. Phys. A: Math. Gen. 37, 2105 (2004).

[29] T. Chou, Biophys. J. 85, 755 (2003).

[30] T. Chou and G. Lakatos, Phys. Rev. Lett. 93, 198101 (2004).

[31] R.K.P. Zia, J.J. Dong and B. Schmittmann, J. Stat. Phys. 144, 405 (2011).

[32] T. Chou and D. Lohse, Phys. Rev. Lett. 82, 3552 (1999).

[33] E. Levine and R.D. Willmann, J. Phys. A: Math. Gen. 37, 3333 (2004).

[34] M. Liu, K. Hawick, S. Marsland and R. Jiang, Physica A 389, 3870 (2010). 
[35] C. A. Brackley, L. Ciandrini and M. C. Romano, J. Stat. Mech. Theor. Exp. (2012) P03002.

[36] P. Greulich, L. Ciandrini, R.J. Allen and M.C. Romano, Phys. Rev. E 85, 011142 (2012).

[37] A. Basu and D. Chowdhury, Phys. Rev. E 75, 021902 (2007).

[38] A. Garai, D. Chowdhury, D. Chowdhury and T.V. Ramakrishnan, Phys. Rev. E 80, 011908 (2009).

[39] L. Ciandrini, I. Stansfield and M.C. Romano, Phys. Rev. E 81, 051904 (2010).

[40] H.S. Kuan and M.D. Betterton, Biophys. J. 110, 2034 (2016).

[41] D. Chowdhury, A. Garai and J.S. Wang, Phys. Rev. E 77, 050902(R) (2008).

[42] D. Oriola, S. Roth, M. Dogterom and J. Casademunt, Nat. Commun. 6, 8025 (2015).

[43] K.E.P. Sugden, M.R. Evans, W.C.K. Poon and N.D. Read, Phys. Rev. E 75, 031909 (2007).

[44] M.R. Evans, Y. Kafri, K.E.P. Sugden and J. Tailleur, J. Stat. Mech. Theor. Expt., P06009 (2011).

[45] Y. Chai, S. Klumpp, M.J.I. Müller and R. Lipowsky, Phys. Rev. E 80, 041928 (2009)

[46] M. Ebbinghaus and L. Santen, J. Stat. Mech.: Theor. Expt. P03030 (2009).

[47] M. Ebbinghaus, C. Appert-Rolland and L. Santen, Phys. Rev. E 82, 040901 (R) (2010).

[48] S. Muhuri and I. Pagonabarraga, Phys. Rev. E 82, 021925 (2010).

[49] I. Neri, N. Kern and A. Parmeggiani, Phys. Rev. Lett. 107, 068702 (2011).

[50] I. Neri, N. Kern and A. Parmeggiani, Phys. Rev. Lett. 110, 098102 (2013).

[51] I. Neri, N. Kern and A. Parmeggiani, New J. Phys. 15, 085005 (2013).

[52] A.I. Curatolo, M.R. Evans, Y. Kafri and J. Tailleur, J. Phys. A: Math. Theor. 49, 095601 (2016).

[53] S. Klein, C. Appert-Rolland and M.R. Evans, J. Stat Mech. 093206 (2016).

[54] A. Parmeggiani, T. Franosch and E. Frey, Phys. Rev. E 70, 046101 (2004).

[55] I.R. Graf and E. Frey, Phys. Rev. Lett. 118, 128101 (2017).

[56] J. Dong, S. Klumpp and R.K.P. Zia, Phys. Rev. Lett. 109, 130602 (2012).

[57] P. Greulich and A. Schadschneider, Physica A 387, 1972 (2008).

[58] M. Barma, Physica A 372, 22 (2006).

[59] A.B. Kolomeisky, J. Phys. A: Math. Gen. 311153 (1998).

[60] P. Pierobon, M. Mobilia, R. Kouyos and E. Frey, Phys. Rev. E 74, 031906 (2006).

[61] R.J. Harris, R.B. Stinchcombe, Phys. Rev. E 70, 016108 (2004).

[62] C. Enaud, B. Derrida, Europhys. Lett. 66, 83 (2004).

[63] J. Schmidt, V. Popkov and A. Schadschneider, Europhys. Lett., 110, 2 (2015).

[64] B. Embley, A. Parmeggiani and N. Kern, Phys. Rev. E 80, 041128 (2009).

[65] M. E. Foulaadvand, A. B. Kolomeisky and H. Teymouri, Phys. Rev. E 78, 061116 (2008).

[66] P. Greulich and A. Schadschneider, J. Stat. Mech. P04009 (2008).

[67] N. Sarkar and A. Basu, Phys. Rev. E 90, 022109 (2014).
[68] T. Banerjee, A. K. Chandra and A. Basu, Phys. Rev. E 92, 022121 (2015).

[69] I. Dhiman and A. K. Gupta, J. Comp. Phys. 309, 227 (2016).

[70] B. Daga, S. Mondal, A. K. Chandra, T. Banerjee and A. Basu, Phys. Rev. E 95, 012113 (2017).

[71] E. N. M. Cirillo, O. Krehel, A. Muntean and R. van Santen, Phys. Rev. E 94, 042115 (2016).

[72] L. J. Cook, J. J. Dong and A. LaFleur, Phys. Rev. E 88, 042127 (2013).

[73] I. Dhiman, A. K. Gupta, Phys. Lett. A 380, 24, 20382044 (2016).

[74] J.J. Dong, B. Schmittmann, R.K.P. Zia, J. Stat. Phys. 128, 1, 21-34 (2007).

[75] M.E. Foulaadvand, S. Chaaboki and M. Saalehi, Phys. Rev. E 75, 011127 (2007).

[76] S. Goldstein and E.R. Speer, Phys. Rev. E 58, 4226 (1998).

[77] M. Ha, J. Timonen and M. den Nijs, Phys. Rev. E 68, 056122 (2003).

[78] H. Hinrichsen and S. Sandow, J. Phys. A: Math. Gen. 30, 2745 (1997).

[79] S.A. Janowsky and, J.L. Lebowitz, Phys. Rev. A 45, 618 (1992).

[80] S.A. Janowsky and J.L. Lebowitz, J. Stat. Phys. 77, 1, 35-51 (1994).

[81] R. Juhász and L. Santen, F. Iglói, Phys. Rev. Lett. 94, 010601 (2005).

[82] R. Juhász and L. Santen, F. Iglói, Phys. Rev. E 74, 061101 (2006).

[83] K.M. Kolwankar and A. Punnoose, Phys. Rev. E 61, 2453 (2000).

[84] J. Krug, Phys. Rev. Lett. 67, 1882 (1991).

[85] J. Krug, Braz. J. Phys. 30, 1, 97-104 (2000).

[86] G. Lakatos, T. Chou and A. Kolomeisky, Phys. Rev. E 71, 011103 (2005).

[87] K. Mallick, J. Phys. A: Math. Gen. 295375 (1996).

[88] G. Schütz, J. Stat. Phys. 71, 3, 471-505 (1993).

[89] G. Tripathy and M. Barma, Phys. Rev. Lett. 78, 3039 (1997).

[90] G. Tripathy and M. Barma, Phys. Rev. E 58, 1911 (1998).

[91] A. Pottmeier, R. Barlovic, W. Knospe, A. Schadschneider and M. Schreckenberg, Physica A 308, 1-4, 471-482 (2002).

[92] J.J. Dong, B. Schmittmann and R.K.P. Zia, Phys. Rev. E 76, 051113 (2007).

[93] A.B. Kolomeisky, Motor proteins and molecular motors, (CRC Press, 2015).

[94] A.E. Firth and I. Brierley J. Gen. Virol. 93, 1385-1409 (2012).

[95] D. Walsh and I. Mohr, Nat. Rev. Microbiol. 9, 860-875 (2011).

[96] H.HT. Au and E. Jan, WIREs RNA 5, 6, 779-801 (2014).

[97] M. Holcik and N. Sonenberg, Nat. Rev. Mol. Cell Bio 6, 318-327 (2005).

[98] I. M. Terenin, V. V. Smirnova, D. E. Andreev, S. E. Dmitriev and I. N. Shatsky, Cell. Mol. Life Sci. 74, 8, 1431-1455 (2016).

[99] I.Brierley, S. Pennell and R.J.C. Gilbert Nature Rev. Microbiol. 5, 598-610 (2007).

[100] A. Wachter, Trends in gent. 30, 5, 172-181 (2014). 


\begin{tabular}{|c|c|c|c|c|c|c|c|}
\hline$i-1$ & $i$ & $i+1$ & $\cdots$ & $\ldots$ & $i+\ell-2$ & $i+\ell-1 \mid$ & $\begin{array}{l}\text { No. of } \\
\text { possible }\end{array}$ \\
\hline 1 & 2 & 2 & 2 & 2 & 2 & 0 & \multirow{2}{*}{2} \\
\hline 1 & 2 & 2 & 2 & 2 & 2 & 1 & \\
\hline 2 & 2 & 2 & 2 & 2 & 0 & 0 & \multirow{3}{*}{3} \\
\hline 2 & 2 & 2 & 2 & 2 & 0 & 1 & \\
\hline 2 & 2 & 2 & 2 & 2 & 1 & 2 & \\
\hline : & : & : & $:$ & : & $:$ & : & \multirow{3}{*}{\} $\begin{array}{l}: \\
:\end{array}$} \\
\hline : & : & : & : & : & : & : & \\
\hline : & : & : & : & : & : & $:$ & \\
\hline 2 & 2 & 0 & 0 & 0 & 0 & 0 & \multirow{3}{*}{$\ell$} \\
\hline : & : & : & : & : & : & : & \\
\hline 2 & 2 & 1 & 2 & 2 & 2 & 2 & \\
\hline 2 & 0 & 0 & 0 & 0 & 0 & 0 & \multirow{3}{*}{$\ell+1$} \\
\hline : & : & : & : & : & : & : & \\
\hline 2 & 1 & 2 & 2 & 2 & 2 & 2 & \\
\hline 0 & 0 & 0 & 0 & 0 & 0 & 0 & \multirow{3}{*}{$\ell+1$} \\
\hline : & : & : & : & : & : & : & \\
\hline 0 & 1 & 2 & 2 & 2 & 2 & 2 & \\
\hline
\end{tabular}

FIG. 12. Schematic diagram of all possible configurations of rods over $\ell+1$ consecutive sites. In their distribution we have followed total exclusion principle. Here, 0,1 and 2 represents the occupational status of any site. If it is " 0 ", then the site is neither occupied nor covered. If it is " 1 ", then it is occupied (i.e. the left most part of the rod is at this site) and if it is " 2 ", then it is covered by a rod.

[101] P.J. Farabaugh, Programmed Alternative Reading of the Genetic Code. Springer (1997).

[102] J.F. Atkins et al, Recoding: Expansion of Decoding Rules Enriches Gene Expression, Springer (2010).

[103] S. Ghosh, T. Bameta, D. Ghanti and D. Chowdhury, J. Stat. Mech. Theor. Expt. 2016, 123501 (2016).

[104] C.J.T. Lewis, T. Pan and A. Kalsotra, Nat. Rev. Mol. Cell Bio. 18, 202-210 (2017).

\section{Appendix A: Calculation of $P(0, \ldots, 0)$}

We consider a system consisting of a long one dimensional lattice of size $L+\ell-1$ and multiple identical rods, each of size $\ell>1$. We define a probability $P(\underbrace{0, \ldots, 0}_{\ell})$ for the consecutive $\ell$ sites being empty (i.e. neither covered nor occupied) simultaneously. The total number $N$ of possible ways to distribute rods over $\ell+1$ consecutive sites (see Fig,12) is given by

$$
\begin{gathered}
N=2+3+\ldots+(\ell-1)+\ell+(\ell+1)+(\ell+1), \\
N=\frac{(\ell-1)(\ell+2)+4(\ell+1)}{2} .
\end{gathered}
$$

Therefore, the probability that all consecutive $\ell$ sites from site $i$ to $i+\ell-1$ are not occupied is,

$$
P(\underbrace{0, \ldots, 0}_{\ell})=\frac{(1-\rho)^{\ell}}{\Theta}
$$

where

$$
\begin{aligned}
\Theta & =\rho^{2}(1-\rho)^{\ell-1}+\rho(1-\rho)^{\ell} \\
& +(\ell-2) \rho(1-\rho)^{\ell} \\
& +\left[\frac{\ell-2}{2}\right] \rho^{2}(1-\rho)^{\ell-1}(\ell+1) \\
& +(1-\rho)^{\ell+1}+\ell \rho(1-\rho)^{\ell} \\
& +\rho(1-\rho)^{\ell}+\ell \rho^{2}(1-\rho)^{\ell-1},
\end{aligned}
$$

is the sum of the weightage of all the possible configurations of the consecutive sites from $i$ to $i+\ell-1$. On further simplification Eq. A3 reduces to

$$
P(\underbrace{0, \ldots, 0}_{\ell})=\frac{2(1-\rho)}{2(1-\rho)(1+\rho(\ell-1))+\rho \ell(2+\rho(\ell-1))} .
$$

Next, we assume that inside each of the segments I, II and III the number density of the rods is uniform and that it is $\rho_{1}, \rho_{2}$ and $\rho_{3}$ in the segments I, II and III, respectively. Although this is a very good approximation in LD and HD phases, it is not so good in the MC phase. If special site is at $i=i_{s}=L_{2}+n \ell+m$ and $n>0$, then,

$$
P(\underbrace{0, \ldots, 0}_{\ell})=\frac{2\left(1-\rho_{3}\right)}{2\left(1-\rho_{3}\right)\left(1+\rho_{3}^{\prime}\right)+\rho_{3} \ell\left(2+\rho_{3}^{\prime}\right)},
$$

where, $\rho_{3}^{\prime}=\rho_{3}(\ell-1)$.

\section{For LD phase in segment III}

$$
\rho_{3}=\alpha_{e f f 3}, \quad 1-\rho_{3}=1-\alpha_{e f f 3},
$$

$P(\underbrace{0, \ldots, 0}_{\ell})=\frac{2\left(1-\alpha_{e f f 3}\right)}{2\left(1-\alpha_{e f f 3}\right)\left(1+\alpha_{e f f 3}^{\prime}\right)+\alpha_{e f f 3} \ell\left(2+\alpha_{e f f 3}^{\prime}\right)}$,

where, $\alpha_{\text {eff } 3}^{\prime}=\alpha_{\text {eff } 3}(\ell-1)$.

For HD phase in segment III

$$
\begin{gathered}
\rho_{3}=1-\beta, \quad 1-\rho_{3}=\beta, \\
P(\underbrace{0, \ldots, 0}_{\ell})=\frac{2 \beta}{2 \beta\left(1+\beta^{\prime}\right)+(1-\beta) \ell\left(2+\beta^{\prime}\right)},
\end{gathered}
$$

where, $\beta^{\prime}=(1-\beta)(\ell-1)$.

\section{For MC phase in segment III}

$$
\begin{gathered}
\rho_{3}=\frac{1}{2}, \quad 1-\rho_{3}=\frac{1}{2}, \\
P(\underbrace{0, \ldots, 0}_{\ell})=\frac{4}{2(\ell+1)+\ell(\ell+3)} .
\end{gathered}
$$




\section{Appendix B: Phase 2}

$$
\begin{aligned}
\beta= & -s_{0}-0.5 \sqrt{s_{0}-s_{1}-\frac{s_{2}}{s_{3}}-s_{4}} \\
& -0.5 \sqrt{s_{5}-s_{6}+\frac{s_{2}}{s_{3}}+s_{4}-\frac{s_{7}}{s_{8}}}
\end{aligned}
$$

where

$$
\begin{gathered}
s_{0}=\frac{0.25\left(-10 \alpha_{e f f 2}^{2}-11 \alpha_{e f f 2}^{2}-2\right)}{10 \alpha_{e f f 2}^{2}+1} \\
s_{1}=\frac{0.0009\left(6400 \alpha_{e f f 2}^{2}-830 \alpha_{e f f 2}+493\right)}{10 \alpha_{e f f 2}+1} \\
s_{2}=0.005\left(1.014 \times 10^{8} \alpha_{e f f 2}^{4}+6.32 \times 10^{7} \alpha_{e f f 2}^{3}-5.07 \times 10^{6} \alpha_{e f f 2}^{2}-241660 \alpha_{e f f 2}+1.09 \times 10^{6}\right) \\
s_{3}=s_{31}\left(s_{32}+s_{33}\right)^{1 / 3}
\end{gathered}
$$

where,

$$
\begin{gathered}
s_{31}=10 \alpha_{e f f 2}+1 \\
s_{32}=-1.45 \times 10^{15} \alpha_{e f f 2}^{6}-1.37 \times 10^{15} \alpha_{e f f 2}^{5}-2.09 \times 10^{14} \alpha_{e f f 2}^{4}+6.05 \times 10^{13} \alpha_{e f f 2}^{3} \\
+4.51 \times 10^{13} \alpha_{e f f 2}^{2}+1.95 \times 10^{12} \alpha_{e f f 2}-1.64 \times 10^{12} \\
s_{33}=\sqrt{s_{331}+s_{332}+s_{333}} \\
s_{331}=-1 . \times 10^{29} \alpha_{e f f 2}^{12}-1.47 \times 10^{29} \alpha_{e f f 2}^{11}+2.48 \times 10^{29} \alpha_{e f f 2}^{10}+2.95 \times 10^{29} \alpha_{e f f 2}^{9}-1.92 \times 10^{29} \alpha_{e f f 2}^{8} \\
s_{332}=-2.5 \times 10^{29} \alpha_{e f f 2}^{7}-3.73 \times 10^{28} \alpha_{e f f 2}^{6}+1.39 \times 10^{28} \alpha_{e f f 2}^{5}+2.22 \times 10^{27} \alpha_{e f f 2}^{4}-5.22 \times 10^{26} \alpha_{e f f 2}^{3} \\
s_{333}=-1.059 \times 10^{26} \alpha_{e f f 2}^{2}-4.56 \times 10^{24} \alpha_{e f f 2}-8.69 \times 10^{22} \\
s_{4}=s_{41}\left(s_{42}+s_{43}\right)^{1 / 3}
\end{gathered}
$$

where,

$$
\begin{gathered}
s_{41}=\frac{0.00004}{10 \alpha_{e f f 2}+1} \\
s_{42}=s_{32} \\
s_{43}=s_{33} \\
s_{5}=\frac{0.5\left(-10 \alpha_{e f f 2}^{2}-11 \alpha_{e f f 2}^{2}-2\right)^{2}}{\left(10 \alpha_{e f f 2}^{2}+1\right)^{2}}
\end{gathered}
$$




$$
\begin{gathered}
s_{6}=\frac{0.002\left(6400 \alpha_{e f f 2}^{2}-830 \alpha_{e f f 2}+493\right)}{10 \alpha_{e f f 2}+1} \\
s_{7}=0.025\left(s_{71}+s_{72}+s_{73}\right) \\
s_{71}=-\frac{\left(-10 \alpha_{e f f 2}^{2}-11 \alpha_{e f f 2}-2\right)^{3}}{\left(10 \alpha_{e f f 2}+1\right)^{3}} \\
s_{72}=-\frac{0.01\left(2800 \alpha_{e f f 2}^{2}-550 \alpha_{e f f 2}+197\right)}{10 \alpha_{e f f 2}+1} \\
0.01\left(-10 \alpha_{e f f 2}^{2}-11 \alpha_{e f f 2}-2\right)\left(6400 \alpha_{e f f 2}^{2}-830 \alpha_{e f f 2}+493\right) \\
s_{8}=\frac{\left(10 \alpha_{e f f 2}+1\right)^{2}}{\sqrt{s_{0}-s_{1}-\frac{s_{2}}{s_{3}}-s_{4}}}
\end{gathered}
$$

\section{Appendix C: Phase 3}

$$
\alpha_{e f f 2}=\frac{-t_{4}-\sqrt{t_{4}^{2}-4 t_{5} t_{6}}}{2 t_{6}},
$$

where,

$$
\begin{aligned}
t_{4}= & -8 \alpha_{I E} \ell^{3 / 2}-4 \alpha_{I E} \ell^{2}+8 \alpha_{I E} \sqrt{\ell}+4 \alpha_{I E} \\
& -10 \ell^{3 / 2} W_{s}-2 \ell^{5 / 2} W_{s}-\ell^{3} W_{s}+\ell^{3}-6 \ell^{2} W_{s} \\
& +4 \ell^{2}-7 \ell W_{s}-4 \sqrt{\ell} W_{s}-3 \ell-2 W_{s}-2 \\
t_{5} & =2 W_{s}-4 \alpha_{I E} W_{s}-8 \alpha_{I E} \sqrt{\ell} W_{s}+5 \ell W_{s} \\
& -4 \alpha_{I E} \ell W_{s}+\ell^{2} W_{s} \\
& \\
t_{6}= & 10 \ell^{3 / 2}+2 \ell^{5 / 2}+\ell^{3}+6 \ell^{2}+7 \ell+4 \sqrt{\ell}+2 .
\end{aligned}
$$

\section{Appendix D: Phase 4}

$$
\beta_{e f f 2}=\frac{-h_{2}-\sqrt{h_{2}^{2}-4 h_{1} h_{3}}}{2 h_{1}},
$$

where,

$$
\begin{aligned}
h_{1} & =\beta^{3} \ell^{3}-4 \beta^{3} \ell^{2}+5 \beta^{3} \ell-2 \beta^{3}-2 \beta^{2} \ell^{3} \\
& +5 \beta^{2} \ell^{2}-5 \beta^{2} \ell+2 \beta^{2}+\beta \ell^{3}-2 \beta \ell^{2} \\
& +\beta \ell+\ell^{2}+\ell,
\end{aligned}
$$

$$
\begin{aligned}
h_{2} & =-2 \alpha_{I E} \beta^{2} \ell^{2}+4 \alpha_{I E} \beta^{2} \ell-2 \alpha_{I E} \beta^{2}-2 \alpha_{I E} \beta \ell \\
& +2 \alpha_{I E} \beta+\beta^{4}\left(-\ell^{3}\right)+4 \beta^{4} \ell^{2}-5 \beta^{4} \ell \\
& +2 \beta^{4}-\beta^{3} \ell^{3} W_{s}+3 \beta^{3} \ell^{3}+4 \beta^{3} \ell^{2} W_{s}-8 \beta^{3} \ell^{2} \\
& -5 \beta^{3} \ell W_{s}+7 \beta^{3} \ell+2 \beta^{3} W_{s}-2 \beta^{3}+2 \beta^{2} \ell^{3} W_{s} \\
& -3 \beta^{2} \ell^{3}-5 \beta^{2} \ell^{2} W_{s}+4 \beta^{2} \ell^{2}+5 \beta^{2} \ell W s \\
& -\beta^{2} \ell-2 \beta^{2} W_{s}-\beta \ell^{3} W_{s}+\beta \ell^{3}+2 \beta \ell^{2} W_{s} \\
& -\beta \ell W_{s}-\beta \ell-\ell^{2} W_{s}-\ell W_{s},
\end{aligned}
$$

$$
\begin{aligned}
h_{3} & =-2 \alpha_{I E} \beta^{2} \ell W_{s}+2 \alpha_{I E} \beta^{2} W_{s}-2 \alpha_{I E} \beta W_{s} \\
& +\beta^{4}\left(-\ell^{2}\right) W_{s}+3 \beta^{4} \ell W_{s}-2 \beta^{4} W_{s} \\
& +3 \beta^{3} \ell^{2} W_{s}-5 \beta^{3} \ell W_{s}+2 \beta^{3} W_{s}-3 \beta^{2} \ell^{2} W_{s} \\
& +\beta^{2} \ell W_{s}+\beta \ell^{2} W_{s}+\beta \ell W_{s} .
\end{aligned}
$$

$$
\beta_{e f f 1}=\frac{-q_{1}-\sqrt{q_{1}^{2}-4 q_{2} q_{3}}}{2 q_{3}},
$$

where,

$$
\begin{aligned}
q_{1} & =\beta_{\text {eff } 2}^{2} \ell-\beta_{\text {eff } 2}^{2}-\beta_{\text {eff } 2} \ell W_{s}+\beta_{\text {eff } 2} \ell \\
& +\beta_{\text {eff } 2} W_{s}-\beta_{\text {eff } 2}+W_{s}
\end{aligned}
$$

$$
q_{2}=\beta_{e f f 2}^{2}-\beta_{e f f 2} W_{s},
$$

$q_{3}=\beta_{e f f 2}(-\ell)+\beta_{e f f 2}-W_{s}$. 


\section{Appendix E: Phase 7}

where,

$$
\beta=z_{0}-0.5 \sqrt{z_{1}+\frac{z_{2}}{z_{3}}+z_{4}}-0.5 \sqrt{z_{5}-\frac{z_{6}}{z_{7}}-z_{8}-\frac{z_{9}}{z_{10}}} \quad z_{0}=0.75
$$

$$
\begin{gathered}
z_{1}=5.5 \times 10^{-11}\left(-5.2 \times 10^{10}-4.5 \times 10^{9} \alpha_{I E}\right)+2.2+1.8 \times 10^{-11}\left(5.2 \times 10^{10}+4.5 \times 10^{9} \alpha_{I E}\right) \\
z_{2}=2.310^{-11}\left(2.0 \times 10^{19} \alpha_{I E}^{2}+5.4 \times 10^{20} \alpha_{I E}+2.7 \times 10^{20}\right) \\
z_{3}=\sqrt[3]{z_{3}^{+}-\sqrt{z_{3}^{-}}}
\end{gathered}
$$

where,

$$
\begin{gathered}
z_{3}^{+}=1.7 \times 10^{29} \alpha_{I E}^{3}+7.4 \times 10^{30} \alpha_{I E}^{2}+3.2 \times 10^{31} \alpha_{I E}+8.5 \times 10^{30} \\
z_{3}^{-}=4.2 \times 10^{58} \alpha_{I E}^{5}-5.9 \times 10^{60} \alpha_{I E}^{4}-2.4 \times 10^{62} \alpha_{I E}^{3}+1.8 \times 10^{62} \alpha_{I E}^{2}+7.1 \times 10^{61} \alpha_{I E}-6.4 \times 10^{60} \\
z_{4}=1.5 \times 10^{-11} z_{3} \\
z_{5}=4.6-7.4 \times 10^{-11}\left(5.2 \times 10^{10}+4.5 \times 10^{9} \alpha_{I E}\right) \\
z_{6}=2.3 \times 10^{-11}\left(2.0 \times 10^{19} \alpha_{I E}^{2}+5.4 \times 10^{20} \alpha_{I E}+2.7 \times 10^{20}\right) \\
z_{7}=z_{3} \\
z_{8}=1.5 \times 10^{-11} z_{7} \\
z_{9}=0.25\left(-4.5 \times 10^{-10}\left(4.9 \times 10^{8} \alpha_{I E}-1.7 \times 10^{10}\right)-6.7^{-10}\left(4.5 \times 10^{9} \alpha_{I E}+5.2 \times 10^{10}\right)+27.9\right) \\
z_{10}=z_{1}+\frac{z_{2}}{z_{3}}+z_{4}
\end{gathered}
$$

